\title{
TÉCNICAS LÍTICAS DIVERSAS ENTRE LAS SOCIEDADES DE ANTOFAGASTA DE LA SiERRA (PUNA MERIDIONAL ARGENTINA) POSTERIORES A ca. 1100 AP
}

\author{
Alejandra Elías
}

\section{* Introducción}

Resumen

En el período Tardío (ca. 1100-550 AP) en Antofagasta de la Sierra surgieron grupos, emplazados en el fondo de cuenca del río Punilla, con agricultura a gran escala y control político-religioso. Esto no implicó la desaparición de los pastores formativos (ca.3000-1100 AP), siendo diversas las respuestas de estos grupos que habitaron los sectores intermedios de las quebradas subsidiarias del Punilla. Tendencias tecnológicas líticas acompañan la coexistencia de estos grupos determinándose variaciones entre sitios, con fechas posteriores a ca. $1100 \mathrm{AP}$. Asimismo, registramos diferencias entre estos microambientes en la continuidad de estos aspectos entre momentos formativos y tardíos/tardíos-inca. En base a esto, sugerimos que la manufactura y uso de artefactos líticos utilitarios, habrían conformado prácticas materiales con las que estos grupos reprodujeron y recrearon, discursiva y no discursivamente, consciente e inconscientemente, sus límites sociales e identidades.

Palabras claves: período Formativo - período Tardío - período Tardío/ Inca - pastores - élites - técnicas líticas.

During Late Period (ca. 1100-550 BP) in Antofagasta de la Sierra emerged groups emplaced in the bottom of the basin of Punilla

River, with large-scale agriculture and political-religious control. This would not meant the disappearance of formative shepherds ( $c a$. 3000-1100 BP), who developed several answers. They resided in the intermediate sectors of Punilla's subsidiary streams. Lithic technology trends coexist with these social groups and variations in some traits were determined between sites dated after $c a$. $1100 \mathrm{BP}$. Also, some differences between these microenvironments in the continuity of these aspects from formative to late/late-inca times were detected. We suggest that the manufacture and use of utilitarian stone artifacts, conformed material practices through which these groups reproduced and recreated, discursively and not discursively, consciously and unconsciously, their social boundaries and identities.

Key words: Formative period - Late period - Late/Inca period shepherds - elites - lithic techniques.

Recibido: febrero 2013. Aceptado: junio 2013.
En la medida en que avanzaron las investigaciones sobre los conjuntos líticos de sitios arqueológicos con fechas posteriores a ca. 1100 AP de la cuenca de Antofagasta de la Sierra (en adelante, ANS) y su comparación con aquellos relevados en asentamientos correspondientes al período Formativo, entre ca. 3000-1100 AP, empezaron a hacerse evidentes las diferencias en la continuidad de determinados patrones tecnológicos (recursos líticos y minerales utilizados, morfologías de las bases de las puntas de proyectil y frecuencias de determinados grupos y subgrupos tipológicos) entre los distintos microambientes como fondo de cuenca del Punilla y sectores intermedios de sus quebradas subsidiarias (Elías 2010).

Estas diferencias parecían acompañar una propuesta desarrollada a partir de estudios del arte rupestre, que señala que al interior de las sociedades de ANS de momentos tardíos estarían ocurriendo procesos con un fuerte desbalance entre grupos dedicados a la producción pastoril, en los sectores intermedios de la cuenca del río Punilla, y otros orientados a las actividades agrícolas de gran escala y al control político-religioso, en el fondo de la misma cuenca (Aschero 2000; Martel y Aschero 2007; Martel 2009).

En este trabajo exponemos las tendencias tecnológicas registradas en conjuntos líticos relevados en sitios con

\footnotetext{
1 Instituto Nacional de Antropología y Pensamiento Latinoamericaco y CONICET. 3 de Febrero 1378 (C1426BJN), Buenos Aires, ARGENTINA. Email: alejandra.elias2@gmail.com

2 En el Noroeste Argentino corresponde al período de los Desarrollos Regionales.
} 
fechados posteriores a ca. $1100 \mathrm{AP}$, emplazados en el fondo de cuenca del río Punilla y en los sectores intermedios de los ríos Las Pitas y Miriguaca, confrontándolas con la información disponible respecto a la evidencia lítica procedente de sitios formativos emplazados en esos mismos microambientes. Estas tendencias son discutidas desde una concepción del proceso tecnológico lítico como un conjunto de técnicas y productos materiales imbricados no solo en condiciones tecnológicas y materiales particulares, sino también asociados a una comprensión compartida y socialmente contextualizada y atravesados por diversos significados políticos, identitarios y de estatus) (Lechtman 1977; Lemonnier 1986; Dietler y Herbich 1998; Stark 1998).

\section{* El Área de estudio}

La microrregión de ANS forma parte de la Puna argentina, uno de los sectores del extremo meridional del altiplano andino, al sur del lago Titicaca, y corresponde al altiplano surboliviano-argentino-chileno o Puna de Atacama (Olivera y Elkin 1994; Berenguer 2004; Reboratti 2006). Se trata de una planicie ubicada por encima de los $3000 \mathrm{~m} . \mathrm{snm}$ que abarca parte de las provincias de Salta, Jujuy, Catamarca, La Rioja y San Juan (Reboratti 2006). Considerando criterios geográficos y ecológicos puede dividirse en dos grandes sectores: Puna Norte y Puna Meridional (Feruglio 1946).

La Puna Meridional, donde se encuentra la microrregión de ANS, abarca la porción suroccidental de la provincia de Jujuy y las porciones de Puna de las provincias de Salta y Catamarca. Es extremadamente fría y seca, con una red hidrográfica endorreica pobremente organizada debido a la escasez de precipitaciones; su rasgo característico la presencia de grandes salares (Olivera 1992; Albeck 2001).

La microrregión de ANS se halla en el ángulo noroeste de la provincia de Catamarca y en ella se encuentra la cuenca del río Punilla, red hidrográfica muy importante de la Puna Meridional (Tchilinguirian y Barandica 1995). Los ríos Miriguaca, Las Pitas, Ilanco, Los Colorados, Mojones, Toconquis y los arroyos de Curuto tributan al $\mathrm{Pu}$ nilla (Olivera et al. 2004; Tchilinguirian y Olivera 2011) (Figura 1).
En la zona de estudio se han distinguido tres sectores con variaciones ecológicas y topográficas y oferta diferencial de recursos faunísticos, vegetales y minerales: fondo de cuenca (3400-3500 m.snm), sectores intermedios (3550-3800 m.snm) y quebradas de altura (3900-4600 m.snm) (Olivera 1992; Olivera y Podestá 1993).

El primer sector es el que ofrece mejores posibilidades para la agricultura debido a su topografía abierta, suelos y disponibilidad de agua. Las terrazas y la planicie aluvial a lo largo del río Punilla presentan vegas con agua permanente, suelos orgánicos desarrollados sobre materiales finos que retienen alta humedad y capas freáticas someras (Olivera y de Aguirre 1995; Tchilinguirian y Barandica 1995). Los sectores intermedios se emplazan en los cursos inferiores y medios de los afluentes del Punilla. Presentan tierras aptas para la producción agropastoril, con disponibilidad de forraje y agua durante todo el año, aunque con menos extensión que en el primer sector (Olivera 1992; Olivera y Podestá 1993). Finalmente, las quebradas de altura incluyen los cursos medios y superiores de los ríos Las Pitas y Miriguaca. Son sectores de quebradas protegidas y estrechas, con cursos permanentes de agua, vegas con pasturas de alta calidad y presencia de forraje diverso del pajonal de altura, aptos para las actividades pastoriles (Olivera 1992; Olivera y Podestá 1993).

\section{* Complejidad sociopolítica en ans desde ca. $1100 \mathrm{AP}$}

El período comprendido entre ca. 900-1460 DC ha sido caracterizado por ciertos cambios sociales, políticos y económicos en distintas regiones del Noroeste Argentino (NOA) y de la subárea Circumpuneña: crecimiento demográfico, uso más intensivo de los territorios, incremento de la agricultura, regionalización de ciertas materialidades (p.e., cerámica), desarrollo de jerarquías y de élites, y surgimiento de diferentes sistemas sociopolíticos en pugna por el acceso a las tierras productivas (González y Pérez 1993 [1972]; Núñez Regueiro 1974; Núñez y Dillehay 1995 [1979]; Tarragó 2000; Albeck 2001; Nielsen 2001).

En ANS desde ca. 1100/1000 AP se habría agudizado la tendencia (iniciada ca. $2000 \mathrm{AP}$ ) hacia el aumento de la importancia de la producción agrícola en la subsistencia 


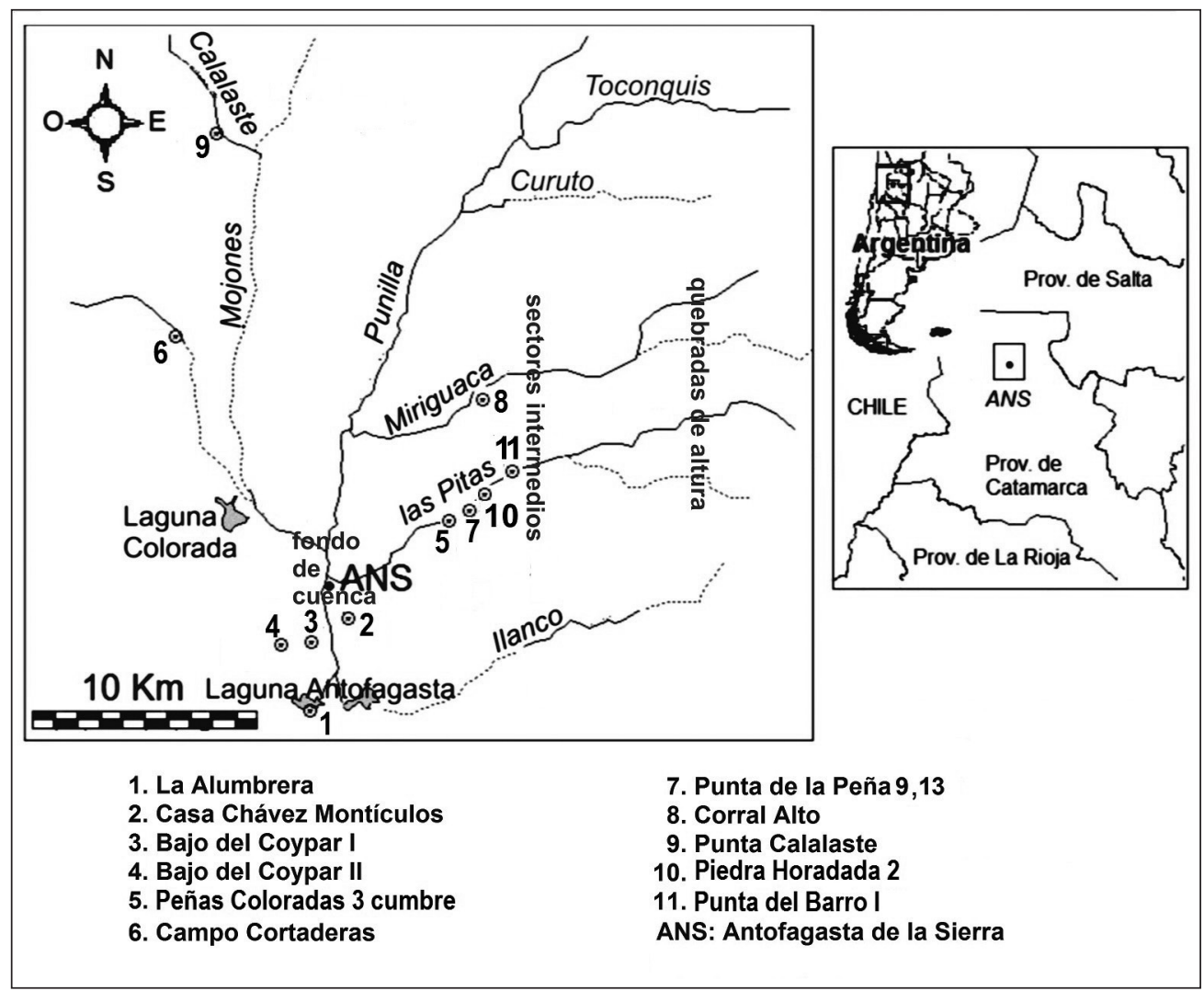

Figura 1. Ubicación de sitios arqueológicos en la microrregión de Antofagasta de la Sierra.

(Olivera y Vigliani 2000-2002). Grandes extensiones de campos de cultivo registradas en diversos sectores de la microrregión corresponderían a estos momentos, como por ejemplo, Bajo del Coypar I, Campo Cortaderas y Punta Calalaste (ver Figura 1) (Olivera y Vigliani 20002002; Olivera et al. 2003-2005; Olivera et al. 2008; Tchilinguirian y Olivera 2011).

Para ca. $700 \mathrm{AP}$ se ha sugerido una creciente concentración y segmentación del poder y surgimiento de élites entre las sociedades de la microrregión (Olivera y Vigliani 2000-2002), aunque tendencias observadas en el arte rupestre de la cuenca llevan a sugerir un escenario aún más complejo. Aschero (2000) señala que, al igual que en otros contextos tardíos de los Andes Centro-Sur, la producción de arte rupestre en ANS adquiere una serie de pautas representacionales particulares: mayor estandarización en los patrones de diseño de ciertos motivos (principalmente camélidos y algunas figuras antropo- morfas) y menor variabilidad en los temas representados. Este autor asocia esto a procesos que se estarían dando al interior de las sociedades, relacionados con un fuerte desbalance entre grupos dedicados a la producción pastoril frente a otros orientados a las actividades agrícolas de gran escala y al control político-religioso. Los últimos corresponderían a jefaturas o sociedades corporativas que estarían acrecentando su poder en términos de control de espacios productivos y fortaleciéndose a través de la reiteración de estilos locales (Aschero 2000; Martel y Aschero 2007; Martel 2009).

Este fortalecimiento de las élites o grupos corporativos explicaría, asimismo, cambios observados en la distribución de los paneles con arte rupestre de la microrregión. Los sitios con representaciones tardías se hacen más frecuentes en el fondo de cuenca del Punilla (Confluencia, Derrumbes, Casas Viejas), lo que podría estar respondiendo a la reestructuración de los espacios 
productivos por parte de la élite y a la significación de nuevos lugares para administrar el nuevo ritual asociado a ésta (Martel 2009).

En tal sentido, son también llamativas las disposiciones de ciertos motivos, escutiformes, túnicas y maquetas, en paneles con arte rupestre de diversos sectores de ANS (Aschero et al. 2009). Los dos primeros se hallan generalmente superpuestos a motivos preexistentes, ocupando lugares vacíos de los paneles o ubicados en sectores altos de los mismos, probablemente en relación a situaciones de imposición iconográfica (Aschero 2000; Martel y Aschero 2007; Martel 2009). Por ejemplo, en el panel superior del sitio Confluencia (fondo de cuenca del río Punilla) se registraron un escutiforme y dos grandes túnicas superpuestas al resto de los grabados (Martel 2009). Otro caso es el escutiforme con anfisbena de Peñas Coloradas I (sectores intermedios del río Las Pitas), el que fue agregado al panel con representaciones de modalidades rupestres anteriores y cuya ejecución se realizó con una técnica diferente (Martel y Aschero 2007).

En lo que refiere a las maquetas tardías, frecuentemente se encuentran en soportes muy visibles lo que las diferencia de aquellas asignadas a contextos formativos previos (modalidad Peñas Chicas), ejecutadas en soportes de baja visibilidad en sectores intermedios. Esto último podría asociarse a un uso discrecional de estas representaciones, dirigido a un entorno de observadores más reducido, es decir, unidades familiares específicas con derechos de uso sobre ese espacio particular del paisaje. En cambio, la ubicación de las maquetas tardías podría interpretarse, en el marco de una estrategia agrícolapastoril con diferenciación social y laboral compleja, en función de un acceso no restricto a un amplio número de observadores (Aschero et al. 2009).

Resumiendo, las tendencias registradas para el arte rupestre tardío de la microrregión estarían relacionadas a una nueva forma de concebir y percibir el espacio y a nuevas normas en la administración del ritual, los espacios destinados a este fin y sus ejecutores. Estas nuevas formas y normas se habrían desarrollado en el escenario de los profundos cambios de la organización social y política de las sociedades puñenas, desde un sistema basado en la familia nuclear como unidad económica y la familia ex- tensa como eje de interacción social a distancia, hacia el surgimiento de jefaturas o sociedades corporativas, que habrían sustraído fuerza de trabajo y bienes del trabajo o producción cooperativo inter e intra-familiar (Martel y Aschero 2007; Martel 2009).

Ahora bien, ¿qué pasó con las antiguas unidades familiares en esta nueva coyuntura de creciente concentración de poder y recursos por parte de determinados grupos (élites, grupos corporativos)? Martel y Aschero (2007) plantean que las nuevas situaciones no habrían necesariamente llevado a la desaparición de los pastores y las respuestas del imaginario de este mundo interfamiliar y pastoril frente a las nuevas presiones habrían sido diversas. El paso de un modo de producción doméstico o cooperativo interfamiliar a uno basado en las demandas de fuerza de trabajo y/o recursos por parte de las élites en surgimiento, habría constituido un punto de conflicto y tensión social. Los autores plantean que si bien aún no sabemos cómo podrían haberse dado las relaciones entre familias y élites, las evidencias etnohistórica y etnográfica indican que esos ejes económicos interfamiliares habrían continuado en funcionamiento, operando como unidades básicas de producción del componente pastoril de las nuevas formas económicas y sociales (Aschero 2000; Martel y Aschero 2007). Cabe señalar que parte importante del arte rupestre tardío se encuentra en soportes con representaciones rupestres preexistentes, emplazados en espacios de explotación económica en la periferia más alejada del poblado fortificado de La Alumbrera (en adelante LA) y próximos a asentamientos de reducida extensión (caseríos, asentamientos aislados, abrigos rocosos) en los sectores intermedios, es decir, en el hábitat de los campesinos y pastores. Esto señala una situación compleja, en la que se mantiene, por un lado, la proximidad a los espacios de actividad doméstica y producción pastoril, y por otro, cierta cercanía a los poblados mayores con posibilidades de defensa y protección. En este sentido, también corresponde mencionar la disposición en los soportes de los conjuntos de camélidos de perfil asignados a momentos tardíos. En los distintos sitios donde se registraron (Punta del Pueblo, Peñas Coloradas 2, Cueva Cacao IA y Peñas Coloradas 3) aparecen generalmente superpuestos a motivos preexistentes, como mascariformes o figuras humanas de brazos alzados (Aschero 2000). Esto podría asociarse a la vigencia de esos íco- 
nos en los cultos locales y al mantenimiento de esos cultos domésticos en torno a la fertilidad y multiplicación de los rebaños de camélidos por parte de las unidades familiares pastoras (Martel y Aschero 2007).

\section{* Estilo material y estilo tecnológico}

La conformación de grupos, límites sociales e identidades son aspectos de la práctica social, situacional y continuamente generados y construidos por las personas, discursiva y no discursivamente, consciente e inconscientemente. La cultura material es activamente usada por las personas y se encuentra involucrada en sus prácticas sociales (Stark 1998; Emberling 1999; Lucy 2005).

La identificación de grupos y límites sociales generalmente ha sido abordada en arqueología desde el "estilo material", es decir, los rasgos formales de los restos dejados por las sociedades pasadas (p.e., cerámica decorada) y su distribución en amplios espacios (Dietler y Herbich 1998; Stark 1998; Lucy 2005). Ahora bien, dentro de la antropología y la arqueología, se desarrollaron posturas que consideraron las tareas y técnicas del proceso de producción tecnológico, del que estos restos son productos, en plena relación con las del quehacer social de los individuos que las llevan adelante (Leroi-Gourhan 1943, 1945; Mauss 1967, 1979; Lechtman 1977; Lemonnier 1986; Pfaffenberger 1992; Stark 1998; Lucy 2005). Desde estas perspectivas, el conocimiento implicado en la creación y uso de los artefactos es constituido en mundos sociales e históricos y es contextualmente específico, sin necesidad de ser plasmado discursivamente. Los actos técnicos son loci claves en los cuales se producen y reiteran los significados culturales. Las elecciones técnicas a lo largo del proceso de manufactura de diversas piezas son consideradas acciones socialmente contextualizadas basadas en una comprensión compartida de cómo las cosas son hechas (Lechtman 1977; Lemonnier 1986; Stark 1998). La tecnología (prácticas técnicas y productos materiales) es un "fenómeno social total", al mismo tiempo material, social, político y simbólico. Estos aspectos confluyen no solo en las formas y los "estilos materiales" (Dietler y Herbich 1998), sino también en el "modo de hacer", las elecciones técnicas a lo largo del proceso productivo, lo que se ha denominado "estilo de acción" (Dietler y
Herbich 1998), "estilo tecnológico" (Lechtman 1977) o "sistema técnico" (Lemonnier 1986).

Esta concepción de la tecnología ha abierto la posibilidad de incorporar los artefactos no decorados (utilitarios) en el estudio de los grupos, límites sociales e identidades (Stark 1998). La manufactura y uso de artefactos líticos, al igual que otros aspectos de la cultura material, constituyen prácticas materiales llevadas adelante por las personas en contextos sociales e históricos específicos y por las que se producen, reproducen y negocian los significados sociales (Dobres 2000; Silliman 2003).

En este marco, y como un primer acercamiento, nos proponemos explorar las variaciones en la tecnología lítica registrada en asentamientos del fondo de cuenca y sectores intermedios de ANS correspondientes a momentos previos y posteriores a ca. $1100 \mathrm{AP}$.

\section{* Metodología de Campo y de laboratorio}

Haremos una breve descripción de los sitios arqueológicos posteriores a ca. $1100 \mathrm{AP}$, de los que proceden los conjuntos líticos analizados. Estos se emplazan en el fondo de cuenca del río Punilla y en los sectores intermedios de algunas de sus quebradas subsidiarias. En el primer grupo, se consideró el asentamiento de LA. Los sitios correspondientes al segundo grupo son: Peñas Coloradas 3 cumbre (en adelante $\mathrm{PC}_{3}$ ), en los sectores intermedios del río Las Pitas, y Corral Alto (en adelante CA), en los sectores intermedios del río Miriguaca.

LA es un gran sitio residencial ubicado $5-6 \mathrm{~km}$ al sur del poblado actual de ANS. En base a la arquitectura y restos cerámicos (predominantemente Belén y cerámica ordinaria, con presencia de fragmentos Santa María, Inca Provincial y Gris Inciso) habría sido ocupada en los períodos Tardío o de Desarrollos Regionales del NOA, Inca e Histórico. Este asentamiento presenta elementos que lo relacionan a una etapa tardía de la Cultura Belén de los valles de Abaucán y Hualfín, al este de ANS (Raffino y Cigliano 1973; Olivera 1991a; Olivera y Vigliani 2000-2002). Actualmente, se cuenta con cinco fechados radiocarbónicos (Tabla 1). 


\begin{tabular}{|c|c|c|c|c|c|c|c|c|c|}
\hline Microambiente & Sitio & Sector & Recinto & Nivel/Capa & Laboratorio & \begin{tabular}{|c|} 
Código \\
laboratorio \\
\end{tabular} & $\begin{array}{l}\text { Tipo de } \\
\text { muestra }\end{array}$ & Años AP C ${ }^{14}$ & Referencia \\
\hline \multirow{21}{*}{$\begin{array}{c}\text { Fondo de cuenca } \\
\text { río Punilla }\end{array}$} & \multirow{7}{*}{$\mathrm{CCHM}$} & \multirow{6}{*}{ Montículo 1} & \multirow{6}{*}{-} & - & Beta Analytic & B-27199 & Carbón & $1670 \pm 60$ & \multirow{7}{*}{$\begin{array}{c}\text { Escola (2000); } \\
\text { Olivera (1992, } \\
\text { 1991b) }\end{array}$} \\
\hline & & & & - & Beta Analytic & B-27201 & Carbón & $1530 \pm 70$ & \\
\hline & & & & - & Beta Analytic & $\mathrm{B}-27202$ & Carbón & $1740 \pm 60$ & \\
\hline & & & & - & Beta Analytic & B-27200 & Carbón & $1930 \pm 70$ & \\
\hline & & & & - & LATYR & LP-299 & Hueso & $2120 \pm 60$ & \\
\hline & & & & - & LATYR & LP-251 & Carbón & $1660 \pm 60$ & \\
\hline & & Montículo 4 & - & - & Beta Analytic & B-27198 & Carbón & $1740 \pm 100$ & \\
\hline & \multirow{8}{*}{ BCII } & \multirow{4}{*}{ III } & \multirow{4}{*}{$\mathrm{b}$} & - & $\begin{array}{l}\text { Universidad de } \\
\text { Georgia (UGA) }\end{array}$ & 7374 & Carbón & $790 \pm 60$ & \multirow{8}{*}{$\begin{array}{l}\text { Olivera y Vigliani } \\
(2000-2002)\end{array}$} \\
\hline & & & & - & UGA & 7315 & Carbón & $1020 \pm 60$ & \\
\hline & & & & - & UGA & 7375 & Carbón & $700 \pm 60$ & \\
\hline & & & & - & UGA & 8625 & $\begin{array}{c}\text { Restos óseos } \\
\text { humanos }\end{array}$ & $1080 \pm 210$ & \\
\hline & & \multirow{4}{*}{ IV } & \multirow{4}{*}{$\mathrm{b}$} & - & UGA & 7517 & Carbón & $650 \pm 50$ & \\
\hline & & & & - & UGA & 7519 & Carbón & $630 \pm 60$ & \\
\hline & & & & - & UGA & 7520 & Carbón & $660 \pm 60$ & \\
\hline & & & & - & UGA & 7518 & Carbón & $880 \pm 80$ & \\
\hline & \multirow{6}{*}{ LA } & Sin datos & $\begin{array}{c}\text { Tumba } \\
\text { colectiva }\end{array}$ & - & Sin datos & Sin datos & $\begin{array}{c}\text { Restos óseos } \\
\text { humanos }\end{array}$ & $210 \pm 70$ & \multirow{6}{*}{$\begin{array}{c}\text { Olivera y Vigliani } \\
(2000-2002) ; \text { Oli- } \\
\text { vera y cols. }(2008) ; \\
\text { Elías (2010) }\end{array}$} \\
\hline & & Central Este & 1 & - & $\begin{array}{c}\text { NSF-Arizona } \\
\text { AMS Lab }\end{array}$ & AA82552 & $\begin{array}{l}\text { Carbón } \\
\text { vegetal }\end{array}$ & $916 \pm 50$ & \\
\hline & & \multirow{4}{*}{ Central Oeste } & Tumba & - & $\begin{array}{c}\text { NSF-Arizona } \\
\text { AMS Lab }\end{array}$ & AA 82550 & Madera & $534 \pm 59$ & \\
\hline & & & 1 & - & $\begin{array}{c}\text { NSF-Arizona } \\
\text { AMS Lab }\end{array}$ & AA7 8543 & $\begin{array}{l}\text { Carbón } \\
\text { vegetal }\end{array}$ & $981 \pm 39$ & \\
\hline & & & \multirow[b]{2}{*}{2} & - & $\begin{array}{l}\text { NSF-Arizona } \\
\text { AMS Lab }\end{array}$ & AA82551 & $\begin{array}{l}\text { Carbón } \\
\text { vegetal }\end{array}$ & $1007 \pm 50$ & \\
\hline & & & & - & $\begin{array}{c}\text { NSF-Arizona } \\
\text { AMS Lab }\end{array}$ & AA78542 & $\begin{array}{l}\text { Carbón } \\
\text { vegetal }\end{array}$ & $536 \pm 42$ & \\
\hline \multirow{3}{*}{$\begin{array}{c}\text { Sectores } \\
\text { intermedios río } \\
\text { Miriguaca }\end{array}$} & \multirow{3}{*}{$\mathrm{CA}$} & \multirow{3}{*}{ Noreste } & \multirow{3}{*}{1} & - & $?$ & Ua-33241 & $\begin{array}{l}\text { Semilla de } \\
\text { chañar }\end{array}$ & $720 \pm 40$ & \multirow{3}{*}{$\begin{array}{c}\text { Escola y } \\
\text { cols. (2011) }\end{array}$} \\
\hline & & & & - & La Plata & LP-1986 & $\begin{array}{l}\text { Carbón } \\
\text { vegetal } \\
\end{array}$ & $660 \pm 60$ & \\
\hline & & & & - & La Plata & LP-2535 & $\begin{array}{l}\text { Carbón } \\
\text { vegetal }\end{array}$ & $860 \pm 60$ & \\
\hline \multirow{18}{*}{$\begin{array}{c}\text { Sectores } \\
\text { intermedios río } \\
\text { Las Pitas }\end{array}$} & $\mathrm{PC}_{3} \mathrm{C}$ & - & $?$ & - & La Plata & LP-1930 & $\begin{array}{c}\text { Carbón vege- } \\
\text { tal yóseo }\end{array}$ & $850 \pm 60$ & Cohen (2009) \\
\hline & \multirow{11}{*}{$\mathrm{PP}_{9}$} & \multirow{3}{*}{ I } & 2 & 4 & UGA & 15101 & $\begin{array}{c}\text { Carozo de } \\
\text { chañar }\end{array}$ & $1270 \pm 50$ & $\begin{array}{c}\text { López Campeny } \\
(2009)\end{array}$ \\
\hline & & & & - & La Plata & LP-1473 & Carbón & $1410 \pm 70$ & \\
\hline & & & 3 & - & La Plata & LP-1430 & guano & $1430 \pm 60$ & Babot y cols. (2006) \\
\hline & & & & 2 (pasillo) & UGA & 9260 & Carbón & $530 \pm 50$ & \\
\hline & & & & 5 (interior) & UGA & 9070 & Carbón & $1150 \pm 150$ & \\
\hline & & & 2 & 3 (pasillo) & UGA & 9069 & $\begin{array}{l}\text { Semilla de } \\
\text { chañar }\end{array}$ & $1460 \pm 40$ & $\begin{array}{l}\text { López Campeny } \\
\qquad(2001)\end{array}$ \\
\hline & & III & & 6 (interior) & UGA & 9076 & Guano & $1970 \pm 50$ & \\
\hline & & & & 3 & La Plata & LP-1553 & Carbón & $380 \pm 70$ & \\
\hline & & & & $5 \mathrm{C}$ & UGA & 9067 & Carbón & $706 \pm 60$ & Cohen (2005); \\
\hline & & & 3 у 4 & $6 a y b$ & UGA & 9261 & Sin datos & $1290 \pm 50$ & $\begin{array}{l}\text { Somonte y Cohen } \\
(2006)\end{array}$ \\
\hline & & & & $6 \mathrm{~d}$ & UGA & 15106 & Sin datos & $1090 \pm 50$ & \\
\hline & & & & Sin datos & La Plata & LP-1620 & $\begin{array}{l}\text { Carbón } \\
\text { vegetal }\end{array}$ & $580 \pm 60$ & \\
\hline & & & 1 & Sin datos & La Plata & LP-1572 & $\begin{array}{l}\text { Carbón } \\
\text { vegetal }\end{array}$ & $690 \pm 60$ & \\
\hline & $\mathrm{PH}_{2}$ & & 2 & Sin datos & La Plata & LP-1887 & $\begin{array}{l}\text { Carbón } \\
\text { vegetal }\end{array}$ & $1270 \pm 50$ & $\begin{array}{l}\text { López Campeny } \\
(2009)\end{array}$ \\
\hline & & & & 5 & La Plata & LP-1876 & Guano & $1630 \pm 90$ & \\
\hline & & & 5 & 12 & La Plata & LP-1716 & Guano & $1670 \pm 60$ & \\
\hline & & & & 14 & La Plata & LP-1875 & Guano & $1870 \pm 100$ & \\
\hline
\end{tabular}

Tabla 1. Fechados radiocarbónicos de sitios arqueológicos formativos y tardíos/tardíos-inca de ANS.

\footnotetext{
64 \begin{tabular}{l|l}
$N^{\circ} 47 / 2014$ \\
EstUdiOs ATACAMEÑos \\
Arqueología y Antropología
\end{tabular} 
LA se emplaza en las laderas del volcán Antofagasta y sus recintos se hallan construidos en la piedra basáltica producto de las emanaciones del mismo. Su topografía revela áreas altas (crestas) y pequeñas hondonadas (abras). La concentración de las construcciones varía en distintos sectores, presentando carácter más aglutinado hacia el centro del asentamiento (Olivera 1991a). Los conjuntos líticos considerados provienen de sondeos y recolecciones superficiales efectuadas en la cresta y el abra del sector central oeste. ${ }^{3}$ Los primeros se realizaron en los recintos 1 ( $\left.\mathrm{R}_{1} \mathrm{O}\right), 2$ ( $\left.\mathrm{R}_{2} \mathrm{O}\right)$ y 14 (R14) de la cresta. En el R1 O se relevaron 83 piezas líticas, 536 en el R2 O y 284 en el $\mathrm{R} 14$. Las recolecciones de superficie fueron realizadas en tres estructuras ubicadas en la cresta (inmediatamente al suroeste del $\mathrm{R}_{1} \mathrm{O}$ ) y en toda la superficie del abra. En los primeros se recolectaron 1170 piezas líticas y en la última 2659 (Elías 2010).

$\mathrm{PC}_{3} \mathrm{C}$ se encuentra en lo alto de las peñas de ignimbritas a lo largo de ambos márgenes del río Las Pitas. Este sitio se halla conformado por 15 estructuras con características arquitectónicas diferenciales potencialmente asociadas a distintos momentos constructivos (Cohen $2009 \mathrm{Ms}$ ). Los líticos considerados $(\mathrm{n}=214)$ provienen de las excavaciones de las estructuras con pared de piedra I, II, III, V, VIII, XI, XIII y hondonada X, y de recolecciones realizadas en toda la superficie $(n=129)$.

Los tiestos toscos y decorados (negro/rojo y negro/crema) relevados en $\mathrm{PC}_{3} \mathrm{C}$ son diagnósticos del período Tardío y recuerdan en algunos casos a la estilística Belén y Villavil. También se han registrado restos asignables a momentos post-contacto. El fechado radiocarbónico obtenido en una de las estructuras es $850 \pm 60 \mathrm{AP}$ (Cohen $2009 \mathrm{Ms}$ ).

Es interesante destacar que desde $\mathrm{PC}_{3} \mathrm{C}$ se cuenta con un amplio control visual del fondo de cuenca del $\mathrm{Pu}-$ nilla, Punta de la Peña y la quebrada de río Miriguaca, así como de las sendas que unen estas localidades. En el marco del modelo planteado por Martel y Aschero (2007), Cohen (2009 Ms) sugiere que este sitio habría sido una estación de control visual, relacionada a un poder corporativo y de resistencia desde los sectores

\footnotetext{
3 La nueva sectorización de LA y el plano correspondiente se encuentran en Salminci (2009).
}

intermedios al dominio implementado por los grupos del fondo de cuenca del Punilla.

CA se emplaza en una cornisa de un farallón de ignimbrita desde la cual se cuenta con un amplio control visual de la cuenca del río Miriguaca. Para acceder al sitio se debe ascender por una explanada de pendiente moderada, y las estructuras que lo conforman solo son visibles al llegar a la cima. Los recintos se distribuyen en dos sectores arquitectónicos: a) el sector suroeste, contra el farallón ignimbrítico, conformado por un patio semicircular de $360 \mathrm{~m}^{2}$ y delimitado por un muro bajo de pirca seca; y b) el sector noreste, con tres estructuras rectangulares de pirca seca y muros elevados (Escola et al. 2011).

Los conjuntos líticos de CA considerados están constituidos por 326 piezas provenientes de sondeos efectuados en la Estructura 1 (E1) del sector noreste, y por 632 piezas relevados en superficie en el patio. Contamos para esta estructura con tres fechados radiocarbónicos posteriores a ca. 900 AP. Se identificó cerámica Belén negro/rojo y fragmentos santamarianos (algunos posiblemente tricolor) (Gasparotti y Escola 2011).

Respecto al análisis de laboratorio, los conjuntos líticos fueron analizados macroscópicamente considerando numerosas variables de la propuesta técnico-morfológica y morfológica-funcional de Aschero (1975 Ms, 1983 Ms, $2008 \mathrm{Ms}$ ). Sin embargo, en esta oportunidad solo se exponen y discuten las frecuencias en que se registran materias primas líticas y minerales y determinados grupos y subgrupos tipológicos, a saber: palas y/o azadas líticas, "raederas de módulos anchísimos" (sensu Hocsman 2006) y puntas de proyectil. Cabe mencionar que las materias primas líticas fueron categorizadas en función de la ubicación de sus potenciales fuentes en distintos sectores/microambientes de la cuenca de ANS, y que en las puntas de proyectil nos centramos en las características formales de las bases o extremos proximales, siguiendo la propuesta de subgrupos tipológicos de Aschero (1983 Ms, $2008 \mathrm{Ms}$ ).

Antes de continuar, señalaremos algunas características de las palas y/o azadas y raederas de módulos anchísimos registradas en ANS. Las primeras corresponden a instrumentos manufacturados en vulcanita 8 , roca volcánica que se presenta en forma de lajas de distintos 


\begin{tabular}{|c|c|c|c|}
\hline Sectores & Microambiente & Fuentes de aprovisionamiento & Materias primas presentes \\
\hline \multirow{21}{*}{$\begin{array}{l}\text { Sector centro-este de la } \\
\text { microrregión de ANS }\end{array}$} & \multirow{6}{*}{ Quebradas de altura río Las Pitas } & Pampa Oeste & $\begin{array}{c}\text { Vulcanitas } 1 \text { (dominante), } 2,3,4,5 \mathrm{y} 7 \\
\text { (posiblemente) }\end{array}$ \\
\hline & & Pampa Este & Vulcanitas 1 (dominante), 2, 3, 4, 5 y 6 \\
\hline & & Pampa Norte & Vulcanita 1 \\
\hline & & QSZAC & Vulcanita 1 \\
\hline & & Vega de Quebrada Seca & Vulcanita 1 \\
\hline & & Las Trancas (curso superior río Ilanco) & Sílice \\
\hline & \multirow{4}{*}{ Sectores intermedios río Las Pitas } & PPZAC & Vulcanitas 1 (dominante), 5 y 7 \\
\hline & & Farallón Salamanca & Vulcanita 1, cuarcita \\
\hline & & Terraza de Punta de la Peña & Cuarcita, sílice \\
\hline & & Rinconada de las Trampas & Cuarcita \\
\hline & \multirow{2}{*}{ Sectores intermedios río Ilanco } & Cantera Inca de Onix & Vulcanita 2 \\
\hline & & PCzZAC & Vulcanita 2 \\
\hline & \multirow{9}{*}{ Fondo de cuenca río Punilla } & Norte Laguna Colorada & Cuarcita \\
\hline & & La Torre/Punta del Pueblo & Cuarcita \\
\hline & & Confluencia & Cuarcita \\
\hline & & Pista Vieja & Cuarzo \\
\hline & & Campo Farfán & Cuarcita, cuarzo \\
\hline & & Bajo del Coypar II & Cuarcita \\
\hline & & Oeste Volcán Antofagasta & Cuarcita \\
\hline & & Los Negros Sector Norte & Vulcanitas 4 y 8 \\
\hline & & Los Negros Sector Sur & Vulcanita 4 , cuarcita \\
\hline \multirow{2}{*}{$\begin{array}{l}\text { Sector norte de la } \\
\text { microrregión de ANS }\end{array}$} & Drenaje río Punilla & Ídem & Vulcanita 1 \\
\hline & $\begin{array}{c}\text { Confluencia de los ríos Punilla y } \\
\text { Miriguaca }\end{array}$ & Ídem & Cuarcita, cuarzo \\
\hline \multirow{2}{*}{$\begin{array}{l}\text { Sector oeste de la } \\
\text { microrregión de ANS }\end{array}$} & Río Calalaste & Ídem & Vulcanita 1 \\
\hline & Campo Cortaderas & Ídem & Vulcanita 4 CCT, vulcanita 8 CCT, cuarzo \\
\hline Salar de Antofalla & Ona-Las Cuevas & Ídem & Obsidiana Ona \\
\hline Salar del Hombre Muerto & Salar del Hombre Muerto & Ídem & Obsidiana Salar del Hombre Muerto \\
\hline Volcán Cueros de Purulla & Cueros de Purulla & Ídem & Obsidianas Cueros de Purulla a y b \\
\hline Laguna Cavi & Laguna Cavi & Ídem & Obsidianas Laguna Cavi 1 y 2 \\
\hline
\end{tabular}

Tabla 2. Distribución de materias primas líticas en los distintos microambientes de ANS. Modificado de Aschero y colaboradores (2002).

tamaños y grosores y que aflora en los márgenes de las coladas de Los Negros y en el área de Campo Cortaderas (Tabla 2, Figura 2). Estos implementos, formatizados por medio de retalla $y / o$ retoque bifacial marginal, han sido relacionados a acciones de cavado en actividades agrícolas, construcción de tumbas, entre otros (Escola 2000; Pérez 2003).
Las "grandes lascas con retoque" (sensu Escola 2000) o raederas de módulos anchísimos conforman implementos con filos obtenidos por retalla y retoque marginal unifacial, que presentan tamaños considerables (superan los $100 \mathrm{~mm}$ de largo y los $200 \mathrm{~mm}$ de ancho) y se hallan manufacturados mayormente en vulcanita 4 . Muestran similitudes con artefactos de La Ciénaga (valle de 


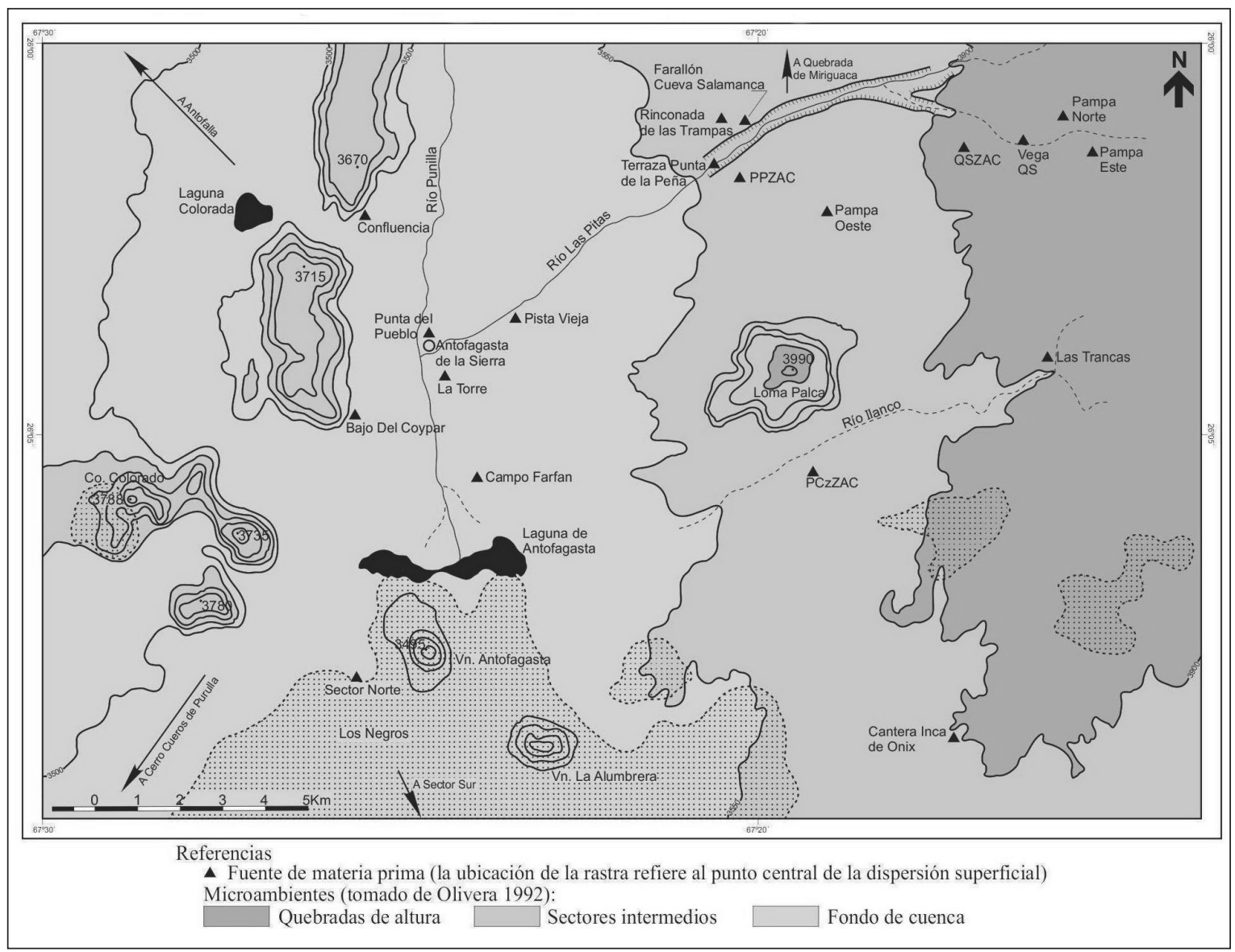

Figura 2. Distribución de fuentes de materias primas líticas en el sector centro-sur de la microrregión de Antofagasta de la Sierra. Tomado de Aschero y colaboradores (2002).

Hualfín) agrupados bajo la denominación de "Industria Basáltica de la Ciénaga" (Menghin 1956 cit. en Escola 2000). Recientes estudios de microfósiles procedentes de estos instrumentos y desechos producto de la reactivación de sus filos, relevados en CCHM y PP9, llevaron a sugerir su uso primario en actividades vinculadas con el manejo de recursos vegetales, particularmente partes útiles de especies agrícolas de ámbitos microtérmicos como pseudocereales domésticos (quinoa y/o cañagua) (Babot et al. 2008).

Ahora bien, acercarse a la complejidad de límites y grupos sociales y sus identidades, requiere documentar la variabilidad práctica y los productos de ésta no solo a través del espacio sino también del tiempo, comparando genealogías o historias de prácticas (Pauketat y Alt 2005). Es por ello que recurrimos a información publicada sobre conjuntos líticos asociados a cronologías previas a ca. 1100 AP correspondientes al período Formativo. Se considerarán, principalmente, las investigaciones realizadas en Casa Chávez Montículos (en adelante CCHM), en el fondo de cuenca del Punilla, y en Punta de la Peña 9 (en adelante PPg) en los sectores intermedios de Las Pitas.

El primer asentamiento ha sido caracterizado como una base residencial de actividades múltiples y se conforma por 10 montículos de origen antrópico. Las excavaciones más importantes se concentraron en los Montículos 1 y 4, registrando evidencias de una larga serie de ocupaciones fechadas entre ca. 2400-1300 AP (ver Tabla 1) y asociadas a diversas actividades (procesamiento y consumo de camélidos, procesamiento de vegetales, manufactura 
de cerámica y talla lítica). En el Montículo 1 se identificaron dos componentes separados por un posible evento de abandono. La abundante presencia de cerámica gris pulida e incisa en el Componente Superior ha llevado a sostener el incremento de la relación con y/o la presencia de grupos provenientes de los valles orientales de Hualfín y Abaucán desde ca. 2000 AP (Olivera 1991b).

Por su parte, $\mathrm{PP} 9$ es una base residencial multicomponente cuya ocupación se inicia hacia 1970 \$50 AP (López Campeny 2001) y continúa hasta el momento colonial moderno (Cohen 2005) (ver Tabla 1). El sitio se halla conformado por recintos subcirculares y elípticos, un área de molienda y bloques con grabados rupestres, dispersos en la terraza alta del río Las Pitas y entre desprendimientos rocosos de farallones de ignimbritas. Se ha determinado que fue utilizado alternativamente a lo largo del tiempo como espacio para múltiples actividades (corrales, áreas de descarte, uso doméstico, actividades rituales) (López Campeny 2001, 2009; Cohen 2005; Babot et al. 2006).

Consideraremos los datos disponibles respecto a los conjuntos líticos relevados en la Estructura 3 (E3) del Sector I de Punta de la Peña 9 (PP9 I) y estructuras 2, 3 y 4 (E2, E3 y E4) del Sector III del mismo sitio (PP9 III). Los fechados de la primera la asocian al período Formativo, mientras que en las estructuras de PPg III no solo se han registrado ocupaciones formativas sino también otras con fechados posteriores a ca. 1100 AP (ver Tabla 1). ${ }^{4}$

Recurriremos también, a estudios de conjuntos líticos procedentes de otros sitios de ANS con fechas anteriores y posteriores a ca. $1100 \mathrm{AP}$, como Punta del Barro I (PBI), estructuras 1, 2 y 5 (E1, E2 y E5) de Piedra Horadada 2 ( $\mathrm{PH} 2)$, Punta de la Peña 13 (PP13), estructuras 2, 6 y 7 (E2, E6 y E7) de PP9 I y Bajo del Coypar II (BCII). Los tres primeros se emplazan en los sectores intermedios del río Las Pitas, y el último en el fondo de cuenca del Punilla. Cabe aclarar que en el caso particular de la quebrada del río Miriguaca aún no contamos con información correspondiente a asentamientos asignables a momentos

\footnotetext{
4 En función de la información disponible para las estructuras de PPy III, consideraremos en bloque las frecuencias de las distintas materias primas líticas, sin discriminar por niveles/capas en los distintos recintos. No desconocemos, sin embargo, la necesidad de evaluar la variación de las frecuencias de las diversas rocas y minerales a lo largo de la estratigrafía.
}

previos a ca. $1100 \mathrm{AP}$, lo que limitó la posibilidad de profundizar en el conocimiento de los cambios diacrónicos de las técnicas líticas.

\section{* Fondo de Cuenca y sectores intermedios DE ANS: DISCONTINUIDAD vs. CONTINUIDAD EN LA TECNOLOGÍA LÍTICA}

\section{Materias primas}

Se observan interesantes diferencias en las frecuencias de diversos recursos líticos de sitios con ocupaciones previas y posteriores a ca. $1100 \mathrm{AP}$ emplazados en el fondo de cuenca del río Punilla y en los sectores intermedios de sus quebradas subsidiarias. En primer lugar, desarrollaremos las tendencias registradas en asentamientos con cronologías posteriores a ca. $1100 \mathrm{AP}$, y luego la información disponible para los sitios con ocupaciones formativas de CCHM y PP9.

En LA se identificaron 23 tipos de rocas y minerales. ${ }^{5} \mathrm{La}$ cuarcita, probablemente proveniente de las fuentes del fondo de cuenca del Punilla (ver Tabla 2 y Figura 2), es la roca predominante en los conjuntos líticos analizados en este sitio (Tabla 3). En esta instancia es importante señalar que en BCII, otro sitio del fondo de cuenca del Punilla con fechados posteriores a ca. 1100 A.P., esta roca también presenta frecuencias dominantes llegando a constituir más del $60 \%$ tanto en los conjuntos de estratigrafía (Escola et al. 2006) como en los de superficie (Elías et al. 2001).

Continuando con LA, cinco son las materias primas identificadas que no se encuentran disponibles en el fondo de cuenca del Punilla, sino que se hallan en fuentes de los sectores intermedios y quebradas altas de los ríos Las Pitas e Ilanco: vulcanitas 1, 2, 3, 7 y sílices translúcidos. Conjuntamente conforman entre el 12-20\% de los conjuntos relevados en este sitio, a excepción del R14 donde solo constituyen el 5.27\%. Son los sílices traslúcidos, con fuentes potenciales en las quebradas altas del Ilanco (ver Tabla 2 y Figura 2), los que hacen que las materias primas provenientes de otros sectores de la cuenca alcancen esas

\footnotetext{
$5 \mathrm{Al}$ considerar el número de rocas y minerales identificadas en los distintos conjuntos líticos no se cuantifica la categoría "no diferenciada".
} 


\begin{tabular}{|c|c|c|c|c|c|c|c|c|c|c|c|c|c|c|c|c|c|c|c|c|c|c|c|c|}
\hline \multirow{3}{*}{$\begin{array}{c}\text { Microambientes } \\
\text { Procedencia } \\
\text { Materias primas }\end{array}$} & \multicolumn{10}{|c|}{ Fondo de cuenca río Punilla } & \multicolumn{10}{|c|}{ Sectores intermedios río Las Pitas } & \multicolumn{4}{|c|}{$\begin{array}{c}\text { Sectores intermedios } \\
\text { río Miriguaca }\end{array}$} \\
\hline & \multicolumn{2}{|c|}{$\mathrm{CCHM}^{\mathrm{a}}$} & \multicolumn{2}{|c|}{$\begin{array}{l}\text { LA } \\
\text { Ri }\end{array}$} & \multicolumn{2}{|c|}{$\begin{array}{l}\mathrm{LA} \\
\mathrm{R} 2\end{array}$} & \multicolumn{2}{|c|}{$\begin{array}{l}\text { LA } \\
\text { Ri4 }\end{array}$} & \multicolumn{2}{|c|}{$\begin{array}{l}\text { LA } \\
\text { sup }\end{array}$} & \multicolumn{2}{|c|}{$\begin{array}{c}\mathrm{PP}_{9} \mathrm{I} \\
\mathrm{E}_{3}{ }^{\mathrm{b}}\end{array}$} & \multicolumn{2}{|c|}{$\begin{array}{l}\mathrm{PP}_{9} \text { III } \\
\mathrm{E}_{2}{ }^{c}\end{array}$} & \multicolumn{2}{|c|}{$\begin{array}{l}\mathrm{PP}_{9} \text { III } \\
\mathrm{E}_{3} \mathrm{yE}_{4}^{\mathrm{d}}\end{array}$} & \multicolumn{2}{|c|}{$\begin{array}{l}\mathrm{PC}_{3 \mathrm{C}} \\
\operatorname{exc}\end{array}$} & \multicolumn{2}{|c|}{$\begin{array}{l}\mathrm{PC}_{3 \mathrm{C}} \\
\sup \end{array}$} & \multicolumn{2}{|c|}{$\begin{array}{l}\text { CA } \\
\text { E1 }\end{array}$} & \multicolumn{2}{|c|}{$\begin{array}{l}\text { CA Patio } \\
\text { sup }\end{array}$} \\
\hline & $\mathrm{n}$ & $\%$ & $\mathrm{n}$ & $\%$ & $n$ & $\%$ & $\mathrm{n}$ & $\%$ & $\mathrm{n}$ & $\%$ & $\mathrm{n}$ & $\%$ & $\mathrm{n}$ & $\%$ & $\mathrm{n}$ & $\%$ & $n$ & $\%$ & $\mathrm{n}$ & $\%$ & $n$ & $\%$ & $\mathrm{n}$ & $\%$ \\
\hline Aragonito & - & - & - & - & 9 & 1.68 & - & - & - & - & - & - & - & - & - & - & - & - & - & - & - & - & - & - \\
\hline Arenisca & 12 & 0.79 & - & - & 2 & 0.37 & - & - & 1 & 0.03 & - & - & - & - & - & - & - & - & - & - & - & - & - & - \\
\hline Carbonato & - & - & - & - & 3 & 0.56 & 4 & 1.4 & 11 & 0.29 & - & - & - & - & - & - & - & - & - & - & - & - & - & - \\
\hline Cuarcita & 44 & 2.89 & 36 & 43.4 & 248 & 46.27 & 162 & 57.04 & 2644 & 69.05 & - & - & 29 & 7.09 & 20 & 1.13 & 5 & 2.34 & 4 & 3.17 & 84 & 25.77 & 145 & 22.94 \\
\hline Cc grano fino & - & - & - & - & 1 & 0.19 & - & - & 1 & 0.03 & - & - & - & - & - & - & - & - & - & - & - & - & - & - \\
\hline cuarzo & 4 & 0.26 & 2 & 2.41 & 42 & 7.84 & 15 & $5 \cdot 3$ & 115 & 3 & - & - & - & - & 6 & 0.34 & 9 & 4.21 & - & - & 6 & 1.84 & 43 & 6.8 \\
\hline Filita & - & - & - & - & - & - & 6 & 2.11 & - & - & - & - & - & - & 35 & 2 & 10 & 4.67 & 2 & 1.6 & 10 & 3.07 & 16 & 2.53 \\
\hline Minerales verdes & - & - & 13 & 15.66 & 73 & 13.62 & 4 & 1.4 & 130 & 3.39 & - & - & - & - & - & - & - & - & - & - & 2 & 0.61 & 3 & 0.47 \\
\hline Obs. CP & 350 & 22.97 & - & - & - & - & 1 & 0.35 & 5 & 0.13 & - & - & 7 & 1.71 & 21 & 1.2 & 4 & 1.86 & - & - & 7 & 2.15 & 9 & 1.42 \\
\hline Obs. LC & - & - & 1 & 1.2 & 2 & 0.37 & 1 & 0.35 & 1 & 0.03 & - & - & - & - & 1 & 0.05 & 1 & 0.47 & - & - & 3 & 0.92 & 5 & 0.8 \\
\hline Obs.nd & - & - & 5 & 6.02 & 2 & 0.37 & 4 & 1.4 & 71 & 1.85 & - & - & - & - & - & - & 6 & 2.8 & - & - & 34 & 10.43 & 41 & 6.49 \\
\hline Obs. O & - & - & 5 & 6.02 & 10 & 1.87 & 8 & 2.82 & 41 & 1.07 & 7 & 4.64 & 37 & 9.05 & 166 & 9.72 & 10 & 4.7 & 3 & 2.38 & 29 & 8.89 & 34 & 5.38 \\
\hline Obs. SHM & - & - & - & - & - & - & - & - & 1 & 0.03 & - & - & - & - & - & - & - & - & - & - & - & - & - & - \\
\hline Sil. traslúcidos & 39 & 2.56 & 15 & 18.07 & 84 & 15.67 & 11 & 3.87 & 383 & 10 & - & - & - & - & - & - & - & - & - & - & 12 & 3.68 & 6 & 0.95 \\
\hline Sil. opacos & - & - & - & - & 2 & 0.37 & 1 & 0.35 & 34 & 0.89 & - & - & - & - & - & - & 1 & 0.47 & - & - & - & - & 4 & 0.63 \\
\hline Sil. otros & 5 & 0.33 & - & - & - & - & - & - & - & - & - & - & 1 & 0.25 & 10 & 0.57 & - & - & - & - & - & - & - & - \\
\hline Vc. 1 & 4 & 0.26 & - & - & 5 & 0.93 & 4 & 1.4 & 22 & 0.57 & 83 & 54.97 & 298 & 72.61 & 799 & 45.98 & 94 & 43.92 & 56 & 44.44 & 53 & 16.26 & 53 & 8.39 \\
\hline Vc. 2 & 55 & 3.61 & 1 & 1.2 & 17 & 3.17 & - & - & 86 & 2.25 & 8 & $5=30$ & - & - & 20 & 1.13 & 14 & 6.54 & 9 & 7.14 & 3 & 0.92 & 14 & 2.22 \\
\hline Vc. 3 & - & - & - & - & 1 & 0.19 & - & - & 1 & 0.03 & 8 & 5.29 & 1 & 0.25 & 23 & 1.31 & 6 & 2.8 & 4 & 3.17 & - & - & 3 & 0.47 \\
\hline Vc. 4 & 468 & 30.71 & 3 & 3.61 & 23 & 4.29 & 44 & 15.5 & 180 & $4 \cdot 7$ & 45 & 29.8 & 33 & 8.06 & 511 & 29.4 & 13 & 6.07 & 17 & 13.5 & 51 & 15.64 & 183 & 28.95 \\
\hline Vc. 5 & 7 & 0.46 & - & - & - & - & - & - & - & - & 1 & 0.66 & - & - & 2 & 0.11 & 17 & 7.94 & 10 & $7 \cdot 94$ & 3 & 0.92 & 6 & 0.95 \\
\hline Vc. 6 & 8 & 0.52 & - & - & - & - & - & - & - & - & - & - & - & - & - & - & 3 & 1.4 & - & - & - & - & - & - \\
\hline Vc. 7 & 137 & 8.99 & - & - & - & - & - & - & 1 & 0.03 & - & - & 2 & 0.49 & 72 & 4.14 & - & - & - & - & 1 & 0.31 & 1 & 0.16 \\
\hline Vc. 8 & 355 & 23.29 & 2 & 2.41 & 7 & 1.31 & 13 & 4.6 & 62 & 1.62 & 7 & 4.64 & 2 & 0.48 & 14 & 0.79 & 1 & 0.47 & - & - & 1 & 0.31 & 1 & 0.16 \\
\hline Vc. nd & - & - & - & - & 5 & 0.93 & 5 & 1.76 & 31 & 0.8 & - & - & - & - & - & - & 10 & 4.67 & 6 & 4.76 & 24 & $7 \cdot 36$ & 44 & 6.96 \\
\hline Vc. vesicular & - & - & - & - & - & - & - & - & 1 & 0.03 & - & - & - & - & - & - & - & - & - & - & - & - & - & - \\
\hline $\mathrm{Nd}$ & - & - & - & - & - & - & 1 & 0.35 & 7 & 0.18 & - & - & - & - & 37 & 2.13 & 10 & 4.67 & 15 & 11.9 & 3 & 0.92 & 21 & 3.33 \\
\hline Otras & 36 & 2.36 & - & - & - & - & - & - & - & - & - & - & - & - & - & - & - & - & - & - & - & - & - & - \\
\hline Totales & 1524 & 100 & 83 & 100 & 536 & 100 & 284 & 100 & 3829 & 100 & 151 & 100 & 410 & 100 & 1737 & 100 & 214 & 100 & 126 & 100 & 326 & 100 & 632 & 100 \\
\hline
\end{tabular}

Tabla 3. Frecuencias de materias primas líticas y minerales en sitios formativos y tardíos/tardíos-inca de ANS. Referencias: Cc: cuarcita; Obs.: obsidiana; Sil.: sílice; Vc.: vulcanita; Nd: no diferenciada; CP: Cueros de Purulla; LC: Laguna Cavi; O: Ona; SHM: Salar del Hombre Muerto; exc: excavación; sup: superficie. ${ }^{a}$ Escola (2000), bBabot y colaboradores. (2006), ' López Campeny (2001), ' $\mathrm{C}$ Cohen (2005) y Somonte y Cohen (2006).

frecuencias. Conforman entre el 10-20\% de los distintos conjuntos líticos del asentamiento, siendo en muchos de éstos, luego de la cuarcita, la materia prima con frecuencias más elevadas. Las rocas provenientes de los sectores intermedios y quebradas altas de Las Pitas no alcanzan ni el 1.5\% (vulcanitas 1, 3 y 7 ) de los conjuntos relevados en LA, o no se hallan representadas (vulcanitas 5 y 6 ).

En $\mathrm{PC}_{3} \mathrm{c}$ se identificaron 16 variedades de rocas y minerales. La vulcanita 1 , disponible en los sectores intermedios y quebradas altas de Las Pitas entre otras localidades (ver Tabla 2 y Figura 2), predomina tanto en estratigrafía como en superficie.

Las materias primas solo disponibles en otros sectores de la microrregión (quebradas altas de Las Pitas, fondo de cuenca del Punilla y/o área de Campo Cortaderas y sectores intermedios del Ilanco) son: vulcanitas 2, 3 , 4 , 6 y 8 y cuarzo (ver Tabla 2 y Figura 2). Conforman conjuntamente el 21-24\% de los conjuntos artefactuales líticos relevados tanto en estratigrafía como excavación. Destacamos que la vulcanita 4 constituye el $6.07 \%$ de los 
conjuntos de excavación y el 13.5\% de los de estratigrafía, y que los recursos líticos de las quebradas altas del río Las Pitas (vulcanitas 3 y 6) y sectores intermedios del Ilanco (vulcanita 2) conjuntamente conforman entre el 9-10\% de ambas muestras.

En CA se identificaron 18 rocas y minerales, 10 de las cuales corresponden a variedades con fuentes potenciales en otros sectores de la microrregión (sectores intermedios y quebradas altas de Las Pitas, fondo de cuenca del Punilla y/o área de Campo Cortaderas, confluencia de los ríos Punilla y Miriguaca, y quebradas altas del río Ilanco): vulcanitas 1, 2, 3, 4, 5, 7 y 8, cuarcita, cuarzo y sílices translúcidos (ver Tabla 2 y Figura 2). Consideradas conjuntamente constituyen entre el 65-72\% de los conjuntos relevados tanto en estratigrafía como en superficie. No se han registrado rocas con fuentes en los sectores intermedios del río Miriguaca, donde se sitúa CA, exceptuando los artefactos de molienda realizados sobre la misma ignimbrita empleada para construirlo.

Las rocas que concentran los porcentajes más elevados en CA son la cuarcita y la vulcanita 4 , aunque ninguna de ellas alcanza a conformar más del 29\% de los conjuntos, como sí ocurre en el caso de la primera variedad en LA y la vulcanita 1 en $\mathrm{PC}_{3}$. La cuarcita de CA posiblemente proceda de una fuente secundaria registrada a $2.5 \mathrm{~km}$ al este de la confluencia de los ríos Punilla y Miriguaca (ver Tabla 2), a menos de $5 \mathrm{~km}$ de CA. Por su parte, la vulcanita 4 podría provenir tanto de los afloramientos del fondo de cuenca del Punilla como de los del área de Campo Cortaderas.

A continuación mencionaremos la representación de distintas materias primas en los conjuntos líticos relevados en los sitios con ocupaciones formativas de CCHM y PP9.

En el Montículo 1 del primer asentamiento, las vulcanitas 4 y 8 son las que concentran los porcentajes más elevados. Los afloramientos de estas rocas más próximos a CCHM, respectivamente, se encuentran en los márgenes de las coladas basálticas de Los Negros y en las de los volcanes Antofagasta y La Alumbrera (ver Tabla 2 y Figura 2). Cabe destacar que pese a que la cuarcita es un recurso ampliamente disponible en el fondo de cuenca del Punilla, donde se emplaza CCHM, solo llega a conformar el $2.89 \%$ del conjunto lítico del Montículo I (Escola 2000).
Las materias primas con fuentes potenciales en otros microambientes son seis (vulcanitas 1, 2, 5, 6 y 7 y sílices traslúcidos -ópalos y calcedonias) y conforman el 16.4\% de la muestra. Las vulcanitas 1, 5, 6 y 7, con fuentes en los sectores intermedios y quebradas altas del río Las Pitas, conforman el $10.23 \%$, con predominio de la última roca (ver Tabla 3).

En PP9 predomina la vulcanita 1 en todas las estructuras, la que posiblemente provenga de la Zona de Aprovisionamiento y Cantera de Punta de la Peña (PPZAC) (ver Tabla 2 y Figura 2).

Respecto de las variedades líticas con fuentes en otros microambientes, son cuatro las identificadas en PP9 I $\mathrm{E}_{3}$ (vulcanitas 2, 3, 4 y 8) y conforman conjuntamente el $39.73 \%$. Este grupo de recursos, compuesto por las vulcanitas 2, 3, 4 y 8 y cuarzo, presenta un porcentaje similar en las $\mathrm{E}_{3}$ y E 4 de PP9 III. Solo en los conjuntos relevados en PPy III E2 estas rocas (vulcanitas 3, 4 y 8) muestran frecuencias menores (8.79\%) (López Campeny 2001). Cabe mencionar que la vulcanita 4 constituye casi el 30\% de los conjuntos de PP9 I E3 y PP9 III E3 y E4 y que las rocas de las quebradas altas del río Las Pitas (vulcanitas 3) y sectores intermedios del Ilanco (vulcanita 2) reúnen conjuntamente bajas frecuencias en los distintos conjuntos, siendo la más elevada de 5.29 \% en PP9 I E3.

\section{Puntas de proyectil}

Sugestivas diferencias se han observado en la continuidad de las morfologías de las bases de las puntas de proyectil registradas en sitios del fondo de cuenca del Punilla y sectores intermedios de Las Pitas y Miriguaca con cronologías anteriores y posteriores a ca. $1100 \mathrm{AP} .^{6}$

Empezando por los asentamientos del fondo de cuenca del Punilla, en los Montículos 1 y 4 de CCHM predominan las puntas de proyectil de limbo triangular, con pedúnculo diferenciado y aletas entrantes. Solo tres puntas de proyectil son de limbo triangular, apedunculadas y base escotada y fueron registradas en los niveles estratigráficos

\footnotetext{
6 Debemos aclarar que análisis más detallados de las características técnico-morfológicas de las puntas de proyectil están en proceso y que las diferencias que planteamos, aunque altamente llamativas, surgen a partir de una caracterización preliminar.
} 


\begin{tabular}{|c|c|c|c|c|c|c|c|c|c|c|c|c|c|c|c|}
\hline \multirow{3}{*}{\multicolumn{2}{|c|}{\begin{tabular}{|c} 
Microambientes \\
Cronología \\
Procedencia/Bases puntas de proyectil
\end{tabular}}} & \multicolumn{7}{|c|}{ Fondo de cuenca río Punilla } & \multicolumn{5}{|c|}{ Sectores intermedios río Las Pitas } & \multirow{2}{*}{\multicolumn{2}{|c|}{$\begin{array}{c}\begin{array}{c}\text { Sectores in- } \\
\text { termedios río } \\
\text { Miriguaca }\end{array} \\
\begin{array}{c}\text { Posteriores a ca. } \\
1100 \mathrm{AP}\end{array}\end{array}$}} \\
\hline & & $\begin{array}{c}\text { Antes de ca. } \\
1100 \mathrm{AP}\end{array}$ & \multicolumn{6}{|c|}{ Posteriores a ca. $1100 \mathrm{AP}$} & \multicolumn{3}{|c|}{ Antes de ca. $1100 \mathrm{AP}$} & \multicolumn{2}{|c|}{$\begin{array}{l}\text { Posteriores a } \\
c a .1100 \mathrm{AP}\end{array}$} & & \\
\hline & & $\mathrm{CCHM}_{1}$ & LA & LA & LA & $\begin{array}{l}\text { BCII } \\
\text { Sector }\end{array}$ & $\begin{array}{l}\text { BCII } \\
\text { Sector }\end{array}$ & BCII & $\mathrm{PP}_{9} \mathrm{I}$ & $\begin{array}{c}\mathrm{PP}_{9} \\
\text { III }\end{array}$ & $\begin{array}{c}\mathrm{PP}_{9} \\
\mathrm{III}\end{array}$ & $\mathrm{PC}_{3} \mathrm{C}$ & $\mathrm{PC}_{3} \mathrm{C}$ & $\mathrm{CA}$ & CA Patio \\
\hline \multirow{3}{*}{ Apedunculadas } & $\begin{array}{l}\text { Apedunculada } \\
\text { con aletas }\end{array}$ & 3 & - & 4 & 1 & 5 & 5 & 6 & - & 1 & 1 & - & - & - & - \\
\hline & $\begin{array}{c}\text { Apedunculada sin } \\
\text { aletas }\end{array}$ & 1 & - & - & - & - & - & - & - & - & - & - & - & - & 2 \\
\hline & $\begin{array}{c}\text { Preforma apedun- } \\
\text { culada }\end{array}$ & - & 1 & - & 1 & - & - & 1 & - & - & - & - & - & - & 2 \\
\hline \multirow{7}{*}{ Con pedúnculo } & $\begin{array}{l}\text { Pedúnculo esbo- } \\
\text { zado con aletas }\end{array}$ & - & - & - & - & - & - & - & - & - & - & - & - & - & 3 \\
\hline & $\begin{array}{l}\text { Pedúnculo desta- } \\
\text { cado con aletas }\end{array}$ & - & - & - & - & - & - & - & - & - & - & - & - & 1 & 1 \\
\hline & $\begin{array}{c}\text { Pedúnculo } \\
\text { diferenciado con } \\
\text { aletas }\end{array}$ & 25 & - & - & - & - & - & - & 1 & 8 & 6 & 2 & 1 & 5 & 9 \\
\hline & $\begin{array}{c}\text { Lancealoda con } \\
\text { pedúnculo y } \\
\text { hombros }\end{array}$ & - & - & - & - & - & - & - & - & 1 & - & - & - & - & - \\
\hline & $\begin{array}{l}\text { Pedúnculo y } \\
\text { aletas, limbo con } \\
\text { módulo geométri- } \\
\text { co romboidal de } \\
\text { tipo equilátero }\end{array}$ & 1 & - & - & - & - & - & - & - & - & - & - & - & - & - \\
\hline & $\begin{array}{l}\text { Preforma pedun- } \\
\text { culada }\end{array}$ & - & - & - & - & - & - & - & - & - & - & - & - & - & 4 \\
\hline & $\begin{array}{l}\text { Fragmento de } \\
\text { pedúnculo }\end{array}$ & - & - & - & - & - & - & - & - & 1 & - & - & - & 1 & - \\
\hline No diferenciada & No diferenciada & 8 & 1 & - & - & 1 & 2 & 1 & - & - & 2 & - & - & 3 & 6 \\
\hline \multicolumn{2}{|l|}{ Totales } & 38 & 2 & 4 & 2 & 6 & 7 & 8 & 1 & 11 & 9 & 2 & 1 & 10 & 27 \\
\hline
\end{tabular}

Tabla 4. Distribución de puntas de proyectil en función de la morfología de sus bases en sitios formativos y tardíos/tardíos-inca de ANS.

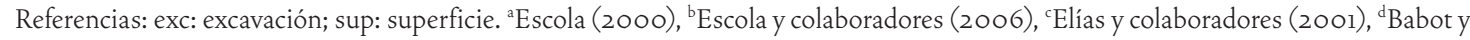
colaboradores (2006), ${ }^{\mathrm{e} L o ́ p e z ~ C a m p e n y ~(2001), ~}{ }^{\mathrm{f}}$ Cohen (2005) y Somonte y Cohen (2006).

datados entre 1740 y $1530 \mathrm{AP}$, correspondientes al Componente Superior (Escola 2000). Por el contrario, en LA gran parte de las puntas de proyectil relevadas corresponden a ejemplares de limbo triangular, apedunculados, con base escotada y aletas. La misma tendencia se observa en BCII (Elías et al. 2001; Escola et al. 2006) (Tabla 4).
En lo que respecta a los sitios de los sectores intermedios de Las Pitas y Miriguaca, en la E3 de PP9 I el único ejemplar de punta de proyectil relevado corresponde a un individuo manufacturado en obsidiana, con limbo triangular isósceles, aletas entrantes y pedúnculo diferenciado (Babot et al. 2006). 
En la E2 de PP9 III se registraron 11 puntas de proyectil. Cuatro ejemplares fueron relevados en los Niveles 3 y 5 , asociados a fechados anteriores a ca. 1100-1000 AP. Dos de ellos presentan pedúnculo y aletas, otro corresponde a un fragmento de pedúnculo, y el cuarto a una punta de limbo lanceolado, pedúnculo destacado y hombros (asociada a una cronología más temprana). Los siete ejemplares restantes fueron obtenidos del Nivel 2, con un fechado de 530 \pm 50 AP (ver Tabla 1). Entre ellos siguen predominando aquellos con pedúnculo y aletas, y solo uno presenta base concavilínea simple (López Campeny 2001).

En la E4 de PP9 III, Cohen (2005) registra nueve puntas de proyectil. Seis de ellas, obtenidas de las capas o, 4 y 5. Presentan pedúnculo diferenciado y aletas y solo una (procedente de la Capa 3 ) es apedunculada, con base escotada y aletas. Las dos restantes corresponden a fragmentos no diferenciados. Cabe mencionar que la Capa 5 cuenta con un fechado de $706 \pm 60 \mathrm{AP}$ (ver Tabla 1), por lo que los ejemplares mencionados corresponderían a contextos posteriores a $1100 \mathrm{AP}$ (Cohen 2005; Somonte y Cohen 2006).

López Campeny (2009) releva cinco puntas de proyectil apedunculadas en el Nivel 3 de la E2 de PPg I, aunque con bases rectas, convexas o cóncavas muy atenuadas. El nivel inmediatamente inferior o 4 posee un fechado de $1270 \pm 50 \mathrm{AP}$ (ver Tabla 1).

Finalmente, en $\mathrm{CA}$ y $\mathrm{PC}_{3} \mathrm{C}$ un importante número de puntas de proyectil corresponden a ejemplares de limbo triangular, con pedúnculo y aletas. En el primer sitio, solo se registraron dos puntas de proyectil apedunculadas sin aletas y dos preformas apedunculadas.

\section{Palas y/o azadas líticas y raederas de módulos anchísimos}

Expondremos las tendencias referidas a las frecuencias de palas y/o azadas líticas, para luego pasar a las raederas de módulos anchísimos. Empezando por los sitios y ocupaciones formativas, en el Montículo 1 de CCHM se identificaron 151 fragmentos de palas y/o azadas líticas, además de conformar la vulcanita 8 una de las variedades de rocas que concentran las porcentajes más elevados entre los desechos de talla (Escola 2000) (Tabla 5).
Fragmentos de palas y/o azadas líticas, junto a desechos de talla en vulcanita 8 , se identificaron también en las E3, E6 y E7 de PP9 I (Babot et al. 2006; López Campeny 2009). No se cuenta con fechados absolutos para las dos últimas estructuras, aunque tentativamente su ocupación no excedería los ca. 1000-1200 AP (López Campeny 2009).

En la E4 de PP9 III se relevó un fragmento de pala y/o azada lítica en la Capa 6d. En 6 a y b, inmediatamente superior, se cuenta con un fechado de $1290 \pm 50 \mathrm{AP}$ (ver Tabla 1) (Cohen 2005; Somonte y Cohen 2006).

Martel (2006, cit. en López Campeny 2009) identifica un ejemplar de pala y/o azada lítica en PP13, asociado a un fechado de $1330 \pm 60$ AP (LP 1585, gramíneas, 600870 cal DC, 2 sigmas).

En la E2 de PHz (ver Tabla 1), López Campeny (2009) releva varios ejemplares de palas y/o azadas líticas en superficie. En la $\mathrm{E}_{5}$ del mismo sitio se registraron tres desechos de vulcanita 8 junto a un fragmento de pala y/o azada lítica en el Nivel 1 (López Campeny 2009).

Finalmente, en PBI se identificaron 18 ejemplares de palas y/o azadas líticas. Si bien aún no se dispone de fechados absolutos, en este asentamiento se han registrado evidencias rupestres y cerámicas que lo asocian a una cronología de ca. 2500-1000 AP (López Campeny 2009).

Ahora mencionaremos las frecuencias en que palas y/o azadas líticas se presentan en asentamientos y ocupaciones correspondientes a momentos posteriores a ca. 1100 AP. Ya hemos señalado (Elías 2006, 2010) que son muy escasos estos artefactos en los conjuntos líticos asignados a esta cronología. Entre los considerados en esta oportunidad, solo se registró un fragmento de este grupo tipológico en las recolecciones de superficie realizadas en LA. Este ejemplar, al igual que sus pares formativos (Escola 2000; Pérez 2003), se encuentra manufacturado en una laja de vulcanita 8 , además de ser evidente su uso prolongado, con filos muy pulidos y redondeados (Elías 2006, 2007).

La casi nula representación de estos objetos se evidencia entre las muestras líticas procedentes de otros sectores 


\begin{tabular}{|c|c|c|c|c|c|c|c|c|c|c|c|c|c|c|c|c|c|c|c|c|c|c|c|c|c|}
\hline \multicolumn{2}{|c|}{$\begin{array}{l}\text { Microam- } \\
\text { bientes }\end{array}$} & \multicolumn{10}{|c|}{ Fondo de cuenca río Punilla } & \multicolumn{10}{|c|}{ Sectores intermedios río Las Pitas } & \multicolumn{4}{|c|}{$\begin{array}{l}\text { Sectores interme- } \\
\text { dios río Miriguaca }\end{array}$} \\
\hline & cedencia & \multicolumn{2}{|c|}{$\mathrm{CCHM}_{1}$} & \multicolumn{2}{|c|}{$\begin{array}{l}\text { LA } \\
\text { Ri }\end{array}$} & \multicolumn{2}{|c|}{$\begin{array}{l}\mathrm{LA} \\
\mathrm{R} 2\end{array}$} & \multicolumn{2}{|c|}{$\begin{array}{l}\text { LA } \\
\text { R14 }\end{array}$} & \multicolumn{2}{|c|}{$\begin{array}{l}\text { LA } \\
\text { sup. }\end{array}$} & \multicolumn{2}{|c|}{$\begin{array}{l}\mathrm{PP}_{9} \mathrm{I} \\
\mathrm{E}_{3}\end{array}$} & \multicolumn{2}{|c|}{$\begin{array}{l}\mathrm{PP}_{9} \mathrm{III} \\
\mathrm{E}_{2}\end{array}$} & \multicolumn{2}{|c|}{$\begin{array}{l}\mathrm{PP}_{9} \mathrm{III} \\
\mathrm{E}_{3} \text { y } \mathrm{E}_{4}\end{array}$} & \multicolumn{2}{|c|}{$\begin{array}{l}\mathrm{PC}_{3 \mathrm{C}} \\
\text { exc. }\end{array}$} & \multicolumn{2}{|c|}{$\begin{array}{l}\mathrm{PC}_{3 \mathrm{C}} \\
\text { sup. }\end{array}$} & \multicolumn{2}{|c|}{$\begin{array}{l}\text { CA } \\
\mathrm{E}_{1}\end{array}$} & \multicolumn{2}{|c|}{$\begin{array}{l}\text { CA Patio } \\
\text { sup. }\end{array}$} \\
\hline \multicolumn{2}{|c|}{$\begin{array}{l}\text { Tipos instru- } \\
\text { mentales y } \\
\text { desechos de } \\
\text { talla }\end{array}$} & $\mathrm{n}^{2}$ & $\%^{\mathrm{b}}$ & $\mathrm{n}$ & $\%$ & $\mathrm{n}$ & $\%$ & $\mathrm{n}$ & $\%$ & $\mathrm{n}$ & $\%$ & $\mathrm{n}^{c}$ & $\%$ & $n^{d}$ & $\%$ & $\mathrm{n}^{\mathrm{e}}$ & $\%$ & $\mathrm{n}$ & $\%$ & $\mathrm{n}$ & $\%$ & $\mathrm{n}$ & $\%$ & $\mathrm{n}$ & $\%$ \\
\hline \multirow{4}{*}{ 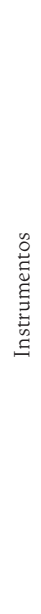 } & $\begin{array}{c}\text { Palas y/o } \\
\text { azadas }\end{array}$ & 151 & 28 & - & - & - & - & - & - & 1 & 0.35 & 3 & 8.33 & 2 & 5.1 & 2 & 3.57 & - & - & - & - & - & - & - & - \\
\hline & $\begin{array}{c}\text { Grandes } \\
\text { lascas } \\
\text { con } \\
\text { retoque }\end{array}$ & 8 & 1.5 & - & - & - & - & - & - & - & - & - & - & - & - & 3 & 5.36 & - & - & - & - & - & - & - & - \\
\hline & $\begin{array}{l}\text { Otros } \\
\text { grupos } \\
\text { tipológi- } \\
\text { cos }\end{array}$ & 372 & 70 & 3 & 100 & 31 & 100 & 11 & 100 & 288 & 99.7 & 33 & 91.7 & 37 & 95 & 51 & 91.1 & 35 & 100 & 42 & 100 & 32 & 100 & 93 & 100 \\
\hline & $\mathrm{n}$ & 531 & 100 & 3 & 100 & 31 & 100 & 11 & 100 & 289 & 100 & 36 & 100 & 39 & 100 & 56 & 100 & 35 & 100 & 42 & 100 & 32 & 100 & 93 & 100 \\
\hline \multirow{4}{*}{ 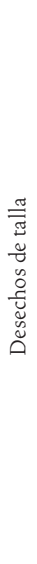 } & Vc. 8 & 2365 & 31 & 2 & 3 & 6 & 1.2 & 13 & 4.76 & 49 & 1.45 & 4 & 1.99 & - & - & 11 & 0.66 & 1 & 0.58 & - & - & 1 & 0.34 & 1 & 0.19 \\
\hline & $\begin{array}{c}\text { Desechos } \\
\text { reactiva- } \\
\text { ción GR }\end{array}$ & sd & $s d$ & $\mathrm{sd}$ & $s d$ & sd & sd & $s d$ & $s d$ & $s d$ & $s d$ & 11 & 5.47 & - & - & 7 & 0.42 & $\mathrm{sd}$ & $\mathrm{sd}$ & $\mathrm{sd}$ & $s d$ & $s d$ & $\mathrm{sd}$ & sd & $\mathrm{sd}$ \\
\hline & $\begin{array}{c}\text { Otras } \\
\text { materias } \\
\text { primas }\end{array}$ & 5210 & 69 & 78 & 97 & 492 & 99 & 260 & 95.2 & 3332 & 98.6 & 186 & 92.5 & 356 & 100 & 1643 & 98.9 & 172 & 99.4 & 84 & 100 & 289 & 99.7 & 529 & 99.8 \\
\hline & $\mathrm{n}$ & 7575 & 100 & 80 & 100 & 498 & 100 & 273 & 100 & 3381 & 100 & 201 & 100 & 356 & 100 & 1661 & 100 & 173 & 100 & 84 & 100 & 290 & 100 & 530 & 100 \\
\hline
\end{tabular}

Tabla 5. Frecuencias de palas y/o azadas líticas y raederas de módulos anchísimos y sus productos secundarios en sitios formativos y tardíos/tardíos-inca de ANS. Referencias: GR: raederas de módulos anchísimos o grandes lascas con retoque; exc: excavación; sup: superficie. ${ }^{2}$ Los núcleos se hallan incluidos en los totales considerados por Escola (2000); bos porcentajes correspondientes a CCHM 1, PP9 I $\mathrm{E}_{3}$ y PP9 III E2, $\mathrm{E}_{3}$ y E4, fueron deducidos a partir de los porcentajes disponibles en la bibliografía; 'Babot y colaboradores (2006, 2008); ${ }^{\mathrm{d} L o ́ p e z ~ C a m p e n y ~(2001) ; ~}{ }^{e}$ Somonte y Cohen (2006); sd: información no disponible. 
de los sitios que aún no fueron sometidas a análisis sistemáticos. Por ejemplo, en CA solo una pala y/o azada ha sido identificada entre los conjuntos relevados en superficie en el sector noreste del asentamiento (Elías 2010).

Estas tendencias se ven reforzadas por las bajas frecuencias de desechos de talla de vulcanita 8 , que podrían asociarse a la manufactura y reactivación de estos instrumentos. Los porcentajes más elevados de desechos de talla de esta roca fueron registrados en el R14 de LA, sin llegar a superar el 5\%. Entre los desechos relevados en la Er y en el patio de CA solo se han identificado dos desechos de talla de vulcanita 8. En $\mathrm{PC}_{3} \mathrm{C}$ un único ejemplar de esta roca fue registrado entre los desechos identificados en excavación.

La muy baja representación de palas y/o azadas líticas y los productos de su manufactura es llamativa en LA y CA, al constituir asentamientos caracterizados como bases residenciales y en los que podrían esperarse tareas de formatización y mantenimiento (Pérez 2003). Particularmente en LA, esta tendencia respecto a las palas y/o azadas contrasta ampliamente con su abundancia en la aldea formativa de CCHM. Es interesante mencionar que, también en BCII, especializado en su última ocupación en actividades agrícolas (Olivera y Vigliani 20002002), se registraron muy bajos porcentajes de desechos de talla de vulcanita 8 y ningún ejemplar de pala y/o azada lítica (Elías et al. 2001; Escola et al. 2006).

En lo que respecta a ocupaciones y sitios posteriores a ca. $1100 \mathrm{AP}$ de los sectores intermedios de Las Pitas, en la E4 de PPg III, Cohen (2005) releva un ejemplar de pala y/o azada lítica en la Capa 1; recordemos que la Capa 5 c cuenta con un fechado de $706 \pm 60$ AP y la Capa 3 con uno de $380 \pm 70 \mathrm{AP}$. En la E2 de PP9 III se relevó un pedúnculo de pala y/o azada lítica en el Nivel 1 y un segundo ejemplar en el Nivel 2; para el último se cuenta con un fechado de $530 \pm 50$ AP (López Campeny 2001). Palas y/o azadas líticas fueron también identificadas en la $\mathrm{E} 1$ de $\mathrm{PH} 2$ con fechados radiocarbónicos posteriores a ca. 1100 AP (López Campeny 2009).

A continuación, mencionaremos las frecuencias de las raederas de módulos anchísimos y los desechos producto de la reactivación de sus filos en sitios y ocupaciones formativas de ANS. En los Niveles II y III (ca.
1670 AP) del Montículo I de CCHM se han recuperado un total de ocho piezas, enteras y fracturadas (Escola 2000).

En la E3 de PP9 I, Babot y colaboradores (2006, 2008) registran desechos de reactivación. En la E4 de PP9 III, Cohen (2005) releva un ejemplar entero y otro fracturado en las capas 5 y 6 inferior, respectivamente, y desechos de reactivación en las capas 5, 6 a y b, $6 \mathrm{~d}$ y 8 . Asimismo, registra un desecho de reactivación en la Capa 5 de la E3 del mismo sector (Cohen 2005; Somonte y Cohen 2006).

Por su parte, López Campeny (2009) encuentra tres ejemplares de raederas de módulos anchísimos vinculados a un fechado de $1270 \pm 50$ AP en la E2 de PP9 I. También registra un fragmento en el Nivel 2 de la $\mathrm{E}_{7}$ de $\mathrm{PP}_{9}$ I, cuya ocupación podría asociarse al período Formativo, aunque aún los investigadores no disponen de fechados absolutos (López Campeny 2009).

¿Qué ocurre con estos implementos en contextos posteriores a ca. 1100 AP? Hasta la actualidad, solamente un ejemplar fue registrado en la $\mathrm{E}_{4}$ de PP9 III, más exactamente en la Capa 4, por encima de la Capa 5 c con un fechado de $706 \pm 60 \mathrm{AP}$. Cohen (2005) señala que este hallazgo amplía el rango temporal de registro de las raederas de módulos anchísimos en ANS. En los restantes sitios considerados, LA, CA y $\mathrm{PC}_{3}$ c, raederas de módulos anchísimos no fueron registradas entre los conjuntos líticos analizados, aunque aún resta profundizar en la identificación de los desechos de reactivación de estos instrumentos (Babot et al. 2008) en estos conjuntos. Es interesante señalar que el grupo tipológico de las raederas (sensu Aschero $1983 \mathrm{Ms}$ ), del que las raederas de módulos anchísimos conforman un subgrupo (sensu Hocsman 2006), tiene muy escasa representación en estos conjuntos (Elías 2010).

\section{* Estilos materiales y tecnológicos líticos DIVERSOS EN LAS SOCIEDADES DE ANS POSTERIORES A CA. 1100 AP}

En este trabajo desarrollamos la variabilidad formal y técnica observada entre conjuntos líticos relevados en sitios con fechados posteriores a ca. 1100 AP emplazados en distintos sectores de ANS y la comparamos con las ten- 
dencias registradas en conjuntos líticos formativos. La información expuesta sugiere que los grupos que habitaron el fondo de cuenca del río Punilla luego de ca. 1100 AP, parecen haber experimentado cambios significativos en sus estilos tecnológicos y materiales líticos respecto a sus antecesores formativos y que esto no parece replicarse en los sectores intermedios de los afluentes de ese curso fluvial. Hasta el momento, son dos los aspectos de la tecnología lítica que muestran llamativas diferencias en lo que refiere a su continuidad entre momentos formativos y tardíos/tardíos-inca en distintos sectores de ANS: materias primas líticas y formas de las bases de las puntas de proyectil. En lo que respecta a las palas y/o azadas líticas y raederas de módulos anchísimos, los cambios más absolutos en sus frecuencias se observan en sitios del fondo de cuenca del Punilla.

Estas tendencias son muy sugestivas a la luz de las investigaciones y propuestas desarrolladas por Martel y Aschero (2007), quienes desde el arte rupestre sugieren para momentos tardíos un escenario de copresencia (conflicto/tensión social) de dos grupos en la microrregión: las élites o grupos corporativos que surgen en el fondo de cuenca del río Punilla, orientados a las actividades agrícolas de gran escala y al control político-religioso, y grupos de pastores, en los sectores intermedios de las quebradas subsidiarias, basados en el antiguo modo de producción doméstico o cooperativo interfamiliar. A continuación, las discutiremos no solo en el marco de esta propuesta, sino considerando también otros aportes.

Empezando por las materias primas representadas, se registran rotundas variaciones en las frecuencias en que ciertas variedades fueron utilizadas por los grupos que habitaron el fondo de cuenca del Punilla entre momentos anteriores y posteriores a ca. $1100 \mathrm{AP}$.

En primer lugar, mientras que en CCHM predomina la vulcanita 4 y la cuarcita muestra muy baja frecuencia, en LA es ésta la roca dominante. La preeminencia de esta variedad en el último asentamiento no constituye un hecho aislado, ya que en BCII, emplazado también en el fondo de cuenca del Punilla y con fechados posteriores a ca. $1100 \mathrm{AP}$, la cuarcita presenta frecuencias dominantes (Elías et al. 2001; Escola et al. 2006). Si bien fuentes de vulcanita 4 y cuarcita se encuentran disponibles en el fondo de cuenca del Punilla, las de la primera roca se ha- llan a más de $5 \mathrm{~km}$ y las de la segunda a menos de $2 \mathrm{~km}$ desde los sitios mencionados.

En segundo lugar, si bien el grupo de recursos con fuentes en otros microambientes de ANS presenta frecuencias similares en CCHM y LA, destacan diferencias interesantes en la representación de algunas variedades de rocas procedentes de determinados sectores. En LA son los sílices traslúcidos, disponibles en las quebradas altas del río Ilanco, los que dominan entre las rocas con fuentes potenciales en otros sectores de la cuenca. Las rocas provenientes de los sectores intermedios y quebradas altas de Las Pitas encuentran muy escasa representación (vulcanitas 1, 3 y 7) o no fueron registradas (vulcanitas 5 y 6 ). Esto es muy diferente de lo que ocurre en CCHM donde los recursos de los sectores intermedios y quebradas altas de Las Pitas constituyen casi el 11\%, predominando la vulcanita 7. Asimismo, la vulcanita 2 con fuentes en los sectores intermedios del río Ilanco presenta porcentajes algo más elevados en CCHM que en LA.

Pareciera que los grupos que habitaron el gran conglomerado de LA accedieron menos frecuentemente que los formativos de CCHM a distintos microambientes de ANS e hicieron un amplio uso de rocas inmediatamente disponibles en el fondo de cuenca del Punilla. Estos cambios en las prácticas tecnológicas líticas de momentos previos y posteriores a ca. 1100 AP podrían ser entendidos en el contexto de otros experimentados por las poblaciones que habitaron este microambiente. Por un lado, es en el fondo de cuenca del Punilla donde, hacia momentos posteriores a ca. $1100 \mathrm{AP}$, habrían empezado a adquirir mayor protagonismo grupos orientados a las actividades agrícolas a gran escala (Aschero 2000; Olivera y Vigliani 2000-2002). Probablemente, esto habría requerido mayor permanencia en este microambiente por parte de una porción importante de los habitantes de LA (así como los de BCII) en las cercanías de las amplias superficies de cultivo de BCI (Tchilinguirian y Olivera 2011) y un acceso menos frecuente a otros sectores de la cuenca y recursos disponibles en ellos. Por su parte, los habitantes de la aldea formativa de CCHM, en el marco de una dinámica pastoril más extendida o generalizada entre las personas, habrían accedido en forma más frecuente a rocas con fuentes más lejanas disponibles en otros sectores de la cuenca (Olivera 1992; Escola 2000). 
Tampoco descartamos que el acceso a determinados recursos haya sido más restricto luego de ca. $1100 \mathrm{AP}$, teniendo en cuenta el escenario de conflicto/tensión sugerido por Martel y Aschero (2007). Los grupos que habitaron el fondo de cuenca del Punilla habrían visto limitado o disminuido el acceso a las fuentes de materias primas líticas de los sectores intermedios y quebradas altas del río Las Pitas. Como discutiremos más adelante, lo mismo ocurriría a los habitantes de los sectores intermedios respecto a los recursos disponibles en el fondo de cuenca del Punilla.

En lo que se refiere a los conjuntos líticos formativos (PP9) y tardíos/tardíos-inca ( $\mathrm{PC}_{3 \mathrm{C}}$ ) de los sectores intermedios de Las Pitas, destaca la recurrencia en el uso predominante de la vulcanita 1 , disminución en $\mathrm{PC}_{3} \mathrm{C}$ respecto a $\mathrm{PP} 9$ del grupo de materias primas con fuentes en otros sectores de la microrregión, y un incremento de las frecuencias de recursos líticos de las quebradas altas del río Las Pitas y sectores intermedios del Ilanco en $\mathrm{PC}_{3}$. Aunque no descartamos que estos cambios se asocien con un incremento del componente agrícola en la subsistencia y una mayor fijación a la tierra, lo que habría implicado un acceso menos frecuente por parte de los habitantes de $\mathrm{PC}_{3} \mathrm{C}$ a otros sectores de la cuenca, es pertinente evaluar otras consideraciones. Por un lado, tengamos en cuenta que los menores porcentajes de recursos con fuentes en otros microambientes de ANS registrados en $\mathrm{PC}_{3}$ c están asociados a una contribución mucho menor de la vulcanita 4 , proveniente probablemente de los afloramientos del fondo de cuenca del Punilla. Por otro lado, que las materias primas con fuentes en las quebradas altas del río Las Pitas (vulcanitas 3 y 6 ) y sectores intermedios del Ilanco (vulcanita 2) presentan porcentajes más elevados en $\mathrm{PC}_{3}$. Estas tendencias nos llevan a preguntarnos si la menor representación en $\mathrm{PC}_{3} \mathrm{C}$ respecto a PPg de rocas con fuentes en otros microambientes fuera de los sectores intermedios del río Las Pitas podría ser también entendida en relación a variaciones en las posibilidades de acceso a distintos sectores de la microrregión por parte de los grupos que habitaron el primer asentamiento, más restricto al fondo de cuenca del río Punilla y más abierto a las quebradas altas del río Las Pitas y sectores intermedios del Ilanco.

Ahora bien, al comparar LA con $\mathrm{PC}_{3} \mathrm{C}$ se observa que en el gran conglomerado del fondo de cuenca del Punilla, los recursos líticos y minerales con fuentes en las quebradas altas del río Las Pitas y sectores intermedios del Ilanco muestran menores porcentajes. Nuevamente, estas diferencias podrían ser entendidas en el marco de una dinámica pastoril más ubicua entre los habitantes de $\mathrm{PC} 3$ c y/o un acceso menos restricto a esos microambientes (ver supra). Como ya mencionamos, una proporción importante de los habitantes de LA, orientados a actividades agrícolas de gran escala, habrían requerido mayor permanencia en las cercanías de los campos de cultivo del fondo de la cuenca del Punilla, accediendo menos frecuentemente a los recursos disponibles en otros sectores. En este contexto, la dinámica pastoril de uso de diversos sectores de la cuenca probablemente no se hallaría ampliamente extendida entre los habitantes del fondo de cuenca del río Punilla luego de ca. 1100 AP.

En LA solo una materia prima con fuentes en otros sectores de ANS presenta porcentajes elevados. Nos referimos a los sílices traslúcidos, utilizados casi exclusivamente en la manufactura de perforadores, implementos con importante presencia en el conjunto instrumental (Elías 2010). Posiblemente, las tareas llevadas a cabo con los perforadores (imanufactura de cuentas?) habrían ocupado un lugar importante en el abanico de actividades desarrolladas por los habitantes del gran conglomerado y esto habría justificado el traslado al curso superior del Ilanco para la obtención de los sílices, conformado recursos que recibieron un tratamiento diferencial por parte de los grupos?

Finalmente, resta destacar la alta frecuencia que en CA, en comparación con LA y $\mathrm{PC}_{3}$, alcanzan los recursos con fuentes en microambientes fuera de los sectores intermedios del Miriguaca, donde se emplaza este asentamiento, y que no se observa predominio de una materia prima específica. Si bien aquellas con fuentes en el fondo de cuenca del Punilla y/o el área de Campo Cortaderas y confluencia de los ríos Punilla y Miriguaca presentan las

\footnotetext{
7 Si bien aún no se han relevado numerosas cuentas en LA, lo que puede estar relacionado a la limitada superficie excavada y sesgos en las muestras, sí se han registrado considerables frecuencias de minerales verdes. En una primera instancia, sobre estas materias primas se habrían manufacturado las cuentas, aunque determinaciones minerológicas detalladas, tanto de los desechos de minerales verdes como de cuentas relevadas en distintos asentamientos posteriores a ca. $1100 \mathrm{AP}$ de ANS, están siendo desarrolladas.
} 
frecuencias más elevadas, junto a los recursos provenientes de otras áreas fuera de la microrregión (p.e., obsidianas), ninguna constituye más del $30 \%$ de los conjuntos, como sí ocurre con la cuarcita en LA y la vulcanita 1 en $\mathrm{PC}_{3} \mathrm{C}$.

Estas tendencias podrían estar relacionadas con la no disponibilidad de rocas adecuadas para ser utilizadas por los talladores en las proximidades de este sitio. Asimismo, el importante registro de recursos procedentes de diversos microambientes de la cuenca nos lleva a sugerir un acceso frecuente a estos últimos por parte de los habitantes de CA. Como en el caso de PC3c, esto podría entenderse inicialmente en el marco de una importante movilidad pastoril y asociarse a la no desaparición de las unidades familiares pastoriles en los sectores intermedios de las quebradas subsidiarías del Punilla (Aschero 2000; Martel y Aschero 2007), aunque futuras investigaciones en la quebrada del Miriguaca contribuirán a una evaluación más estricta de la continuidad/discontinuidad en las elecciones de recursos líticos y minerales y otros aspectos de la tecnología lítica.

En lo que se refiere a las puntas de proyectil, en los sitios emplazados en el fondo de cuenca del río Punilla se observa un cambio desde el predominio de puntas de proyectil con pedúnculo y aletas en contextos formativos (CCHM) hacia el predominio de ejemplares apedunculados, con base escotada y aletas en contextos posteriores a ca. $1100 \mathrm{AP}$ (LA, BCII). Esta discontinuidad no se contesta en los sectores intermedios, donde el uso predominante de puntas de proyectil con pedúnculo y aletas parece continuarse entre momentos formativos y tardíos/ tardíos-inca. Esta última afirmación se sustenta primordialmente en el caso de los sectores intermedios de Las Pitas para la que contamos con datos correspondientes a estos distintos períodos, aunque en los sectores intermedios del Miriguaca cabe subrayar el alto porcentaje que en contextos posteriores a ca. $900 \mathrm{AP}(\mathrm{CA})$ alcanzan los ejemplares con pedúnculo y aletas.

Un último punto a destacar respecto a las puntas de proyectil relevadas en asentamientos posteriores a $c a .700$ AP en ANS es que la convivencia de ejemplares con pedúnculo y aletas y apedunculados se aparta de la tendencia señalada, hasta el momento, por otros investigadores hacia el amplio predominio de puntas apedunculadas con base escotada (sobre las pedunculadas con aletas) en sitios tardíos e incas de otras regiones del NOA (Nielsen 2001; Ávalos 2002; Ledesma 2003; Sprovieri 2005; Chaparro 2009; Flores y Wynveldt 2009, entre otros).

Respecto de las palas y/o azadas líticas, el cambio más rotundo en la representación de estos ejemplares y de los desechos de su manufactura y/o reactivación entre momentos formativos y tardíos/tardíos-inca, se observa en el fondo de cuenca del río Punilla. Estos artefactos muestran una importante presencia en CCHM, en tanto que en LA y BCII se caracterizan por su baja a nula representación. Esto es llamativo, por un lado, en el contexto de la sugerida importancia alcanzada por la agricultura entre los grupos tardíos/tardíos-inca (Olivera y Vigliani 2000-2002) y teniendo en cuenta, además, que el incremento del componente agrícola propuesto luego de ca. 2000 AP en ANS sí coincidió con un aumento de las frecuencias de estos implementos en CCHM (Olivera y Podestá 1993; Olivera y Grant 2008). Asimismo, no debemos olvidar que afloramientos de vulcanita 8 se encuentran en el fondo de la cuenca del Punilla y muy cercanos a LA y BCII, por lo que la roca necesaria para la manufactura de las palas y/o azadas líticas habría estado ampliamente accesible para los habitantes de estos dos últimos asentamientos.

Previamente (Elías 2006, 2007), hemos sugerido que otras materias primas, más específicamente la madera, empezaron a adquirir relevancia en la manufactura de estos artefactos hacia momentos más tardíos. Palas de madera de heterogéneas formas y características fueron registradas en diversos sitios arqueológicos del NOA y en la región del río Loa, Chile (Boman 1992 [1908]; Latcham 1938; Núñez 1974; Uribe et al. 2004). En áreas cercanas a ANS, debemos mencionar un ejemplar en el sitio Campo de Carrizal (valle de Hualfín) correspondiente al período Tardío (Valencia et al. 2009). Ahora bien, la madera con las propiedades necesarias para manufacturar estos instrumentos es un recurso no disponible en la Puna, y probablemente las poblaciones de ANS accedieron a la misma por medio de contactos de larga distancia con grupos de la subárea Valliserrana u otras regiones donde este material estuviera disponible. No obstante, éstas no son más que algunas ideas y conjeturas, ya que aún no se han registrado ejemplares de palas de madera que permitan contrastarlas. 
Los cambios observados en las frecuencias de palas y/o azadas líticas en el fondo de cuenca del Punilla no parecen suceder entre sitios formativos y tardíos/ tardíos-inca de los sectores intermedios, considerando principalmente los datos obtenidos por otros investigadores en asentamientos del río Las Pitas, como PP9 y PHz. Sin embargo, no dejamos de considerar la muy escasa presencia que estos implementos muestran en los conjuntos líticos relevados en los sitios de sectores intermedios analizados en este aporte, $\mathrm{PC}_{3}$ с y CA. Nos preguntamos si razones asociadas a las características de ocupación de estos asentamientos ayudarían a explicar estas tendencias.

Finalmente, en lo que atañe a las raederas de módulos anchísimos destaca su ausencia en los sitios con ocupaciones posteriores a ca. $1100 \mathrm{AP}$ del fondo de cuenca del Punilla, así como el registro de un ejemplar asociado a un fechado tardío en $\mathrm{PP}_{9}$ (Cohen 2005). Esto es interesante si tenemos en cuenta que estos implementos son, incluso, muy escasos en contextos formativos; recordemos que solo se registraron ocho ejemplares en CCHM (Escola 2000). Queda abierta la pregunta si la raedera de módulo anchísimo registrada en contextos tardíos de PPg estaría remitiendo a la continuidad de ciertas prácticas entre los grupos que habitaron los sectores intermedios de Las Pitas.

Finalmente, sugerimos que los grupos que habitaron el fondo de cuenca del Punilla en momentos posteriores a ca. $1100 \mathrm{AP}$ probablemente habrían compartido modos de hacer sus artefactos líticos un tanto diferentes a los formativos de la aldea de CCHM y a los de sus contemporáneos de los sectores intermedios, principalmente de
Las Pitas, quienes habrían continuado manteniendo ciertos estilos materiales y tecnológicos líticos de momentos formativos.

Estas tendencias llaman la atención sobre el modelo planteado por Martel y Aschero (2007) respecto a la coexistencia en momentos tardíos de unidades interfamiliares pastoriles, en los sectores intermedios de la cuenca, con grupos orientados a las actividades agrícolas de gran escala y asociados al control político-religioso en surgimiento en el fondo de cuenca del río Punilla. Estos autores se basan en el estudio de los estilos y motivos rupestres y su distribución en los paneles y el paisaje.

Por nuestra parte, los resultados que alcanzamos estimulan a tomar también en consideración las técnicas y prácticas asociadas a la manufactura y uso de la tecnología lítica. Es decir, los artefactos líticos, no decorados, mundanos y utilitarios, y las técnicas asociadas a su manufactura y uso, habrían sido parte de las prácticas materiales por medio de las que las sociedades antofagasteñas posteriores a ca. $1100 \mathrm{AP}$ recrearon cotidianamente sus identidades, relaciones y límites sociales.

Agradecimientos Al CONICET por solventar con becas doctoral y postdoctoral la realización de este trabajo. A los doctores Patricia Escola, Daniel Olivera y Lorena Cohen por facilitarme los conjuntos artefactuales líticos considerados y sus correspondientes aportes. A Osvaldo Maida por su colaboración en la transcripción al inglés del resumen. A los evaluadores quienes con sus sugerencias contribuyeron a mejorar el manuscrito. Finalmente, a la población de ANS.

\section{$*$ Referencias citadas}

ALBECK, M. E., 2001. La Puna argentina en los períodos Medio y Tardío. En Historia argentina prehispánica, E. Berberian y A. Nielsen (Eds.), pp. 347-388. Editorial Brujas, Córdoba.

ÁVALOS, J. C., 2002. Sistema de producción lítica de una comunidad tardía de la Quebrada de Humahuaca. Tesis de Licenciatura. Facultad de Humanidades y Ciencias Sociales, Universidad Nacional de Jujuy, San Salvador de Jujuy.
ASCHERO, C., 1975 Ms. Ensayo para una clasificación morfológica de artefactos líticos aplicada a estudios tipológicos comparativos.

1983 Ms. Ensayo para una clasificación morfológica de artefactos líticos. Apéndices A y B.

2000. Figuras humanas, camélidos y espacios en la interacción circumpuneña. En Arte en las rocas. Arte rupestre, menhires y 
piedras de colores en la Argentina, M. Podestá y M. de Hoyos (Eds.), pp. 15-44. Sociedad Argentina de Antropología, Buenos Aires.

2008 Ms. Guía de códigos para caracteres morfológicos y dimensionales.

ASCHERO, C., A. MARTEL y S. LÓPEZ CAMPENY, 2009. El sonido del agua... arte rupestre y actividades productivas. El caso de Antofagasta de la Sierra, Noroeste Argentino. En Crónicas sobre la piedra. Arte rupestre de las Américas, M. Sepúlveda, L. Briones y J. Chacama (Eds.), pp. 257-270. Andros Impresores, Santiago.

ASCHERO, C., P. ESCOLA, S. HOCSMAN y J. MARTíneZ, 2002. Recursos líticos en la escala microrregional Antofagasta de la Sierra, 1983-2001. Arqueología 12: 9-36.

BABOT, P., P. ESCOLA y S. HOCSMAN, 2008. Microfósiles y atributos tecno-tipológicos. Correlacionando raederas de módulo grandísimo con sus desechos de talla de mantenimiento en el Noroeste Argentino. En Matices interdisciplinarios en estudios fitolíticos y de otros microfósiles, A. Korstanje y P. Babot (Eds.), pp. 187-200. BAR International Series S1870, Oxford.

BABOT, P., C. ASCHERO, S. HOCSMAN, M. C. HAROS, L. BARONI y S. URQUIZA, 2006. Ocupaciones agropastoriles en los sectores intermedios de Antofagasta de la Sierra (Catamarca): Un análisis desde Punta de la Peña 9. Comechingonia 9: $57-78$.

BERENGUER, J., 2004. Caravanas, interacción y cambio en el desierto de Atacama. Ediciones Sirawi, Santiago.

BOMAN, E., 1992 [1908]. Antigüedades de la Región Andina de la República Argentina y del desierto de Atacama. Universidad Nacional de Jujuy, San Salvador de Jujuy.

COHEN, L., 2005. Entre guano y arena... ocupaciones recurrentes: Un caso de estudio en el sitio Punta de la Peña 9-III Antofagasta de la Sierra Catamarca. Tesis de Licenciatura. Facultad de Ciencias Naturales e Instituto Miguel Lillo, Universidad Nacional de Tucumán, San Miguel de Tucumán.

2009 Ms. Jerarquías, estrategias de control y su espacialidad. Perspectivas desde el sitio Peñas Coloradas 3-Cumbre (Antofagasta de la Sierra, Catamarca). Informe presentado al CONICET, Buenos Aires.

CHAPARRO, M. G., 2009. El manejo de los recursos líticos en el pasado: Sociedades pre-estatales y estatales en el área Vallise- rrana del Noroeste Argentino (1000-1536 DC). Tesis Doctoral. Facultad de Filosofía y Letras, Universidad de Buenos Aires, Buenos Aires.

DIETLER, M. e I. HERBICH, 1998. Habitus, techniques, style: An integrated approach to the social understanding of material culture and boundaries. En The archaeology of social boundaries, M. Stark (Ed.), pp. 232-263. Smithsonian Institution Press, Washington D.C.

DOBRES, M. A., 2000. Technology and social agency. Blackwell Publishers, Nueva York.

ELÍAS, A., 2006. El estudio de la organización de la tecnología lítica en momentos tardíos (ca. 1000-450 AP) en Antofagasta de la Sierra (Prov. de Catamarca). Tesis de Licenciatura. Facultad de Filosofía y Letras, Universidad de Buenos Aires, Buenos Aires.

2007. Tecnología lítica entre las sociedades de ca. 1000 años AP en Antofagasta de la Sierra (prov. de Catamarca, Puna Meridional argentina). Estudios Atacameños, Arqueología y Antropología Surandinas 33: 59-85.

2010. Sociedades agropastoriles del Noroeste Argentino y tecnología lítica. Evidencia lítica en sitios tardíos (ca. 1100-550 años AP) de Antofagasta de la Sierra (Puna Meridional argentina). Tesis Doctoral. Facultad de Filosofía y Letras, Universidad de Buenos Aires, Buenos Aires.

ELÍAS, A., L. PAULIDES y S. VIGLIANI, 2001. Dos astillas del mismo palo: Observaciones tecnológicas preliminares sobre dos sitios de Antofagasta de la Sierra, prov. de Catamarca. Actas XIV Congreso Nacional de Arqueología Argentina, Universidad Nacional de Rosario, Rosario. En prensa.

EMBERLING, G., 1999. The value of tradition: The development of social identities in early Mesopotamian States. En Material symbols. Culture and economy in prehistory, J. E. Robb (Ed.), pp. 277301. Center for Archaeological Investigations, Southern Illinois University, Carbondale.

ESCOLA, P., 2000. Tecnología lítica y sociedades agropastoriles tempranas. Tesis Doctoral. Facultad de Filosofía y Letras, Universidad de Buenos Aires, Buenos Aires.

ESCOLA, P., A. ELÍAS y L. PAULIDES, 2006. Bajo del Coypar II: Tendencias tecnológicas para el Tardío de Antofagasta de la Sierra (Catamarca). Werkén 8: 5-23. 
ESCOLA, P., S. LÓPEZ CAMPENY, A. MARTEL, A. ROMANO y S. HOCSMAN, 2011. Re-conociendo un espacio en lugar de un paisaje. Andes. En prensa.

FERUGLIO, E., 1946. Los sistemas orográficos de la Argentina. Geografía de la República Argentina (Tomo IV). Sociedad Argentina de Estudios Geográficos, Buenos Aires.

FLORES, M. y F. WYNVELDT, 2009. Análisis tecno-tipológico de los artefactos líticos de la Loma de los Antiguos de Azampay (departamento de Belén, Catamarca). Intersecciones en Antropología 10: 221-235.

GASPAROTTI, L. y P. ESCOLA, 2011. Elecciones tecnológicas en la alfarería. Abordaje preliminar de la cerámica de Corral Alto, Antofagasta de la Sierra (Catamarca). La Zaranda de Ideas 8 (1): 49-64.

GONZÁLEZ, A. R. y J. PÉREZ, 1993 [1972]. Argentina indígena. Visperas de la conquista. Paidós, Buenos Aires.

HOCSMAN, S., 2006. Producción lítica, variabilidad y cambio en Antofagasta de la Sierra, ca. 5500-1500 AP. Tesis Doctoral. Facultad de Ciencias Naturales y Museo, Universidad Nacional de La Plata, La Plata.

LATCHAM, R., 1938. Arqueología de la Región Atacameña. Prensas de la Universidad de Chile, Santiago.

LECHTMAN, H., 1977. Style in technology. Some early thoughts. En Material culture: Styles, organization, and dynamics of technology, H. Lechtman y R. Merril (Eds.), pp. 3-20. West Publishing Co. St. Paul, Minn.

LEDESMA, R., 2003. Diseño de puntas de proyectil. Una vía de análisis alternativo para el estudio de identidad en la Quebrada del Toro. Provincia de Salta, Argentina. Cuadernos de la Facultad de Humanidades y Ciencias Sociales de la Universidad de Jujuy 20: 241-269.

LEMONNIER, P., 1986. The study of material culture today: Toward and anthropology of technical systems. Journal of Anthropological Archaeology 5 (2): 147-186.

LEROI-GOURHAN, A., 1943. Evolution et techniques: L'homme et la matiére. Albin Michel, París.

1945. Evolution et techniques: Milieu et techniques. Albin Michel, París.
LÓPEZ CAMPENY, S., 2001. Actividades domésticas y uso del espacio intrasitio. Sitio Punta de la Peña 9 (Antofagasta de la Sierra, Catamarca). Tesis de Licenciatura. Facultad de Ciencias Naturales e Instituto Miguel Lillo, Universidad Nacional de Tucumán, San Miguel de Tucumán.

2009. Asentamiento, redes sociales, memoria e identidad. Primer milenio de la era Antofagasta de la Sierra, Catamarca. Tesis Doctoral. Universidad Nacional de la Plata, Facultad de Ciencias Naturales y Museo, La Plata.

LUCY, S., 2005. Ethnic and cultural identities. En The archaeology of identity. Approaches to gender, age, status, ethnicity and religion, M. Díaz-Andreu, S. Lucy, S. Babi y D. Edwards (Eds.), pp. 86-109. Routledge, Londres y Nueva York.

MARTEL, A., 2009. Arte rupestre: Construcción y significación del espacio en la Puna Meridional argentina (Antofagasta de la Sierra, Catamarca). En Crónicas sobre la piedra. Arte rupestre de las Américas, M. Sepúlveda, L. Briones y J. Chacama (Eds.), pp. 271280. Andros Impresores, Santiago.

MARTEL, A. y C. ASCHERO, 2007. Pastores en acción: Imposición iconográfica vs. autonomía temática. En Producción y circulación prehispánicas de bienes en el sur andino, A. Nielsen, M. C. Rivolta, V. Seldes, M. Vázquez y P. Mercolli (Eds.), pp. 329-349. Editorial Brujas, Córdoba.

MAUSS, M., 1967. The gift: Forms and functions of exchange in Archaic society. Norton, Nueva York.

1979. Les techniques du corps. En Sociology and psychology: Essays of Marcel Mauss, pp. 97-113. Routledge and Kegan Paul, Londres.

MENGHIN, O., 1956. La industria basáltica de La Ciénaga (provincia de Catamarca). Anales de Arqueología y Etnología XII: 289299.

NIELSEN, A., 2001. Evolución social en la quebrada de Humahuaca (700-1536 DC). En Historia argentina prehispánica, E. Berberián y A. Nielsen (Eds.), pp. 347-388. Editorial Brujas, Córdoba.

NÚÑEZ, L., 1974. La agricultura prehistórica en los Andes Meridionales. Universidad del Norte, Santiago.

NÚÑEZ, L. y T. DILLEHAY, 1995 [1979]. Movilidad giratoria, armonía social y desarrollo en los Andes meridionales: Patrones de tráfico e interacción económica. Universidad Católica del Norte, Antofagasta. 
NÚÑEZ REGUEIRO, V., 1974. Conceptos instrumentales y marco teórico en relación al análisis del desarrollo cultural del Noroeste Argentino. Revista del Instituto de Antropología 5: 169-180.

OLIVERA, D., 1991a. La ocupación inka en la Puna Meridional argentina: Departamento de Antofagasta de la Sierra, Catamarca. Comechingonia 9:31-72.

1991b. El Formativo en Antofagasta de la Sierra (Puna Meridional argentina): Análisis de sus posibles relaciones con contextos arqueológico agroalfareros tempranos del Noroeste Argentino y norte de Chile. Actas del XI Congreso Nacional de Arqueología Chilena, vol. 2, pp. 61-78. Sociedad Chilena de Arqueología, Santiago.

1992. Tecnología y estrategias de adaptación en el Formativo (Agroalfarero Temprano) de la Puna Meridional argentina. Un caso de estudio: Antofagasta de la Sierra (provincia de Catamarca, República Argentina). Tesis Doctoral. Facultad de Ciencias Naturales, Universidad Nacional de La Plata, La Plata.

OLIVERA, D. y M. J. DE AGUIRRE, 1995. Arqueología aplicada a la reactivación de sistemas agrícolas prehispánicos: El aporte interdisciplinario. Hombre y Desierto 9: 337-349.

OLIVERA, D. y D. ELKIN, 1994. De cazadores y pastores: El proceso de domesticación de camélidos en la Puna Meridional argentina. En Zooarqueología de camélidos, vol. 1, pp. 95-124. Grupo de Zooarqueología de Camélidos-GZC, Buenos Aires.

OLIVERA, D. y J. GRANT, 2008. Economía y ambiente durante el Holoceno Tardío (ca. 4500-400) de Antofagasta de la Sierra (Puna Meridional argentina). En Temas de arqueología. Estudios tafonómicos y zooarqueológicos (I), A. Acosta, D. Loponte y L. Mucciolo (Eds.), pp. 99-131. Asociación de Amigos del Instituto Nacional de Antropología y Pensamiento Latinoamericano, Secretaría de Cultura de la Nación, Buenos Aires.

OLIVERA, D. y M. PODESTÁ, 1993. Los recursos del arte: Arte rupestre y sistemas de asentamiento-subsistencia formativos en la Puna Meridional argentina. Arqueología 3: 93-141.

OLIVERA, D. y S. VIGLIANI, 2000-2002. Proceso cultural, uso del espacio y producción agrícola en la Puna Meridional argentina. Cuadernos del Instituto Nacional de Antropología y Pensamiento Latinoamericano 19: 459-481.

OLIVERA, D., P. TCHILINGUIRIAN y L. GRANA, 2004. Paleoambiente y arqueología en la Puna Meridional argentina: Archivos ambientales, escalas de análisis y registro arqueológico. Relaciones de la Sociedad Argentina de Antropología XXIX: 229-247.
OLIVERA, D., S. VIGLIANI, A. ELÍAS., L. GRANA y P. TCHILINGUIRIAN, 2003-2005. La ocupación Tardío-Inka en la Puna Meridional: El sitio Campo Cortaderas. Cuadernos del Instituto Nacional de Antropología y Pensamiento Latinoamericano 20: 257-277.

OLIVERA, D., A. ELÍAS, P. SALMINCI, P. TCHILINGUIRIAN, L. GRANA, J. GRANT y P. MIRANDA, 2008. Nuevas evidencias del proceso sociocultural en Antofagasta de la Sierra. Informe de campaña año 2007. La Zaranda de Ideas 4: 119-140.

PAUKETAT, T. y S. ALT, 2005. Agency in a postmold? Physicality and the archaeology of culture-making. Journal of Archaeological Method and Theory 12 (3): 213-237.

PÉREZ, S., 2003. Experimentación y análisis de microdesgaste de palas y/o azadas líticas de Antofagasta de la Sierra (Catamarca). Tesis de Licenciatura. Facultad de Filosofía y Letras, Universidad de Buenos Aires, Buenos Aires.

PFAFFENBERGER, B., 1992. Social anthropology of technology. Annual Review of Anthropology 21: 491-516.

RAFFINO, R. y M. CIGLIANO, 1973. La Alumbrera: Antofagasta de la Sierra. Un modelo de ecología cultural prehispánica. Relaciones de la Sociedad Argentina de Antropología (NS) VII: 241-258.

REBORATTI, C., 2006. Situación ambiental en las ecorregiones Puna y Altos Andes. En La situación ambiental argentina 2005, A. Brown, U. Martínez Ortiz, M. Acerbi y J. Corcuera (Eds.), pp. 33-39. Fundación Vida Silvestre, Buenos Aires.

SALMINCI, P., 2009. Configuración espacial y organización social: Análisis de acceso en La Alumbrera (período Tardío, Puna Meridional argentina). Arqueología 16: 105-124.

SILLIMAN, S., 2003. Using a rock in a hard place: Native-american lithic practices in colonial California. En Stone tool traditions in the contact era, C. Cobb (Ed.), pp. 127-150. The University of Alabama Press, Tuscaloosa.

SOMONTE, C. y L. COHEN, 2006. Reocupación y producción lítica: Un aporte a la historia ocupacional de los recintos 3 y 4 del sitio agropastoril de Punta de la Peña 9-Sector III (Antofagasta de la Sierra, Catamarca, Argentina). Werkén 9: 135-158.

SPROVIERI, M., 2005. Manejo de recursos y producción lítica en sociedades tardías del valle Calchaquí (Salta). Tesis de Licenciatura. Facultad de Filosofía y Letras, Universidad de Buenos Aires, Buenos Aires. 
STARK, M., 1998. Technical choices and social boundaries in material culture patterning: An introduction. En The archaeology of social boundaries, M. Stark (Ed.), pp. 1-11. Smithsonian Institution Press, Washington D.C.

TARRAGÓ, M., 2000. Chacras y pukara. Desarrollos sociales tardíos. En Los pueblos originarios y la Conquista, M. Tarragó (Comp.), pp. 257-300. Editorial Sudamericana, Buenos Aires.

TCHILINGUIRIAN, P. y M. BARANDICA, 1995. Acontecimientos naturales que favorecieron el asentamiento humano en ambientes de la Puna catamarqueña. Hombre y Desierto 9: 351-352.
TCHILINGUIRIAN, P. y D. OLIVERA, 2011. Agricultura, ambiente y sustentabilidad agrícola en el desierto: El caso Antofagasta de la Sierra (Puna argentina, $26^{\circ} \mathrm{S}$ ). En Arqueología de la agricultura: Casos de estudio en la región andina argentina, A. Korstanje y M. Quesada (Eds.), pp. 102-127. Magna, San Miguel de Tucumán.

URIBE, M., L. ADÁN y C. AGÜERO, 2004. Arqueología de los períodos Intermedio Tardío y Tardío de San Pedro de Atacama y su relación con la cuenca del río Loa. Chungara, Revista de Antropología Chilena 36 (2): 943-956.

VALENCIA, M., N. ZAGORODNY y S. RIVERA, 2009. Análisis de restos de madera del sitio Campo de Carrizal. Darwiniana 47 (2): $260-266$. 